\title{
A new implementation of the infrared flux method using the 2MASS catalogue ${ }^{\star}$
}

\author{
J. I. González Hernández ${ }^{1, \star \star}$ and P. Bonifacio ${ }^{1,2, \star \star}$ \\ 1 GEPI, Observatoire de Paris, CNRS, Université Paris Diderot, Place Jules Janssen, 92190 Meudon, France \\ e-mail: [Jonay.Gonzalez-Hernandez;Piercarlo.Bonifacio]@obspm.fr \\ 2 Istituto Nazionale di Astrofisica - Osservatorio Astronomico di Trieste, via Tiepolo 11, 34143 Trieste, Italy \\ Received 3 September 2008 / Accepted 15 January 2009
}

\section{ABSTRACT}

\begin{abstract}
Context. The effective temperature scale of FGK stars, especially at the lowest metallicities remains a major problem in the chemical abundance analysis of metal-poor stars.

Aims. We present a new implementation of the infrared flux method (IRFM) using the 2MASS catalogue.

Methods. We computed the theoretical quantities in the 2MASS $J H K_{\mathrm{s}}$ filters by integrating theoretical fluxes computed from ATLAS models, and compare them directly with the observed 2MASS $J H K_{\mathrm{s}}$ magnitudes. This is the main difference between our implementation of the IRFM and that of Ramírez \& Meléndez (2005, ApJ, 626, 446, hereafter RM05), since to introduce new stars at the lowest metallicities they transform the 2MASS $J H K_{\mathrm{s}}$ magnitudes into the TCS photometric system. We merge in our sample the stars from Alonso et al. (1996, A\&AS, 117, 227, hereafter AAM96; 1999, A\&AS, 139, 335, hereafter AAM99), and other studies to appropriately cover a wide range of metallicities, ending up with 555 dwarf and subgiant field stars and 264 giant field stars. We derived a new bolometric flux calibration using the available Johnson-Cousins $U B V(R I)_{\mathrm{C}}$ and the $2 \mathrm{MASS} J H K_{\mathrm{s}}$ photometry. We also computed new $T_{\text {eff }}$ versus colour empirical calibrations using our extended sample of stars.

Results. We derived effectives temperatures for almost all the stars in the AAM96 and AAM99 samples and find that our scales of temperature are hotter by $\sim 64 \mathrm{~K}\left(\sigma_{\mathrm{T}}=104 \mathrm{~K}, N=332 \mathrm{dwarfs}\right)$ and $\sim 54 \mathrm{~K}$ with a $\sigma_{\mathrm{T}}=131 \mathrm{~K}(N=202$ giants $)$. The same comparison with the sample of RM05 for stars with $[\mathrm{Fe} / \mathrm{H}]<-2.5$ provides a difference of $\sim-87 \mathrm{~K}\left(\sigma_{\mathrm{T}}=194 \mathrm{~K}, N=12\right.$ dwarf stars $)$ and $\sim 61 \mathrm{~K}$ $\left(\sigma_{\mathrm{T}}=62 \mathrm{~K}, N=18\right.$ giant stars).

Conclusions. Our temperature scale is slightly hotter than that of AAM96 and RM05 for metal-rich dwarf stars but cooler than that of RM05 for metal-poor dwarfs. We have performed an fully self-consistent IRFM in the 2MASS photometric system. For those who wish to use 2MASS photometry and colour-temperature calibrations to derive effective temperatures, especially for metal-poor stars, we recommend our calibrations over others available in the literature. In our implementation we avoid the transformation of the 2MASS $J H K_{\mathrm{s}}$ magnitudes to a different photometric system and thus fully exploit the excellent internal consistency of the 2MASS photometric system.
\end{abstract}

Key words. infrared: stars - stars: abundances - stars: atmospheres - stars: fundamental parameters

\section{Introduction}

The effective temperature is a function of the bolometric flux and the angular diameter according to the equation

$T_{\mathrm{eff}}=\left(\frac{4}{\sigma}\right)^{1 / 4} \theta^{-1 / 2} F_{\mathrm{bol}}^{1 / 4}$

where $\sigma$ is the Stefan-Boltzmann constant, $\theta$ the angular diameter, and $F_{\text {bol }}$ the bolometric flux measured on the surface of the Earth. However, direct measure of angular diameters is restricted to relatively few stars, especially for dwarf stars. Kervella et al. (2004, 2008, interferometry) and Brown et al. (2001, transit observations) have directly measured the angular diameters of bright stars. Only recently, Baines et al. (2008) have used the CHARA interferometric array to provide measurements of

* Tables as described in Sect. 3 are only available in electronic form at the CDS via anonymous ftp to

cdsarc.u-strasbg.fr $(130.79 .128 .5)$ or via

http://cdsweb.u-strasbg.fr/cgi-bin/qcat?J/A+A/497/497

$\star \star$ Cosmological Impact of the First STars (CIFIST) Marie Curie Excellence Team. angular diameters of $~ 28$ dwarf and subgiant stars, although all of them have metallicities $[\mathrm{Fe} / \mathrm{H}]>-0.5$.

A semi-direct method of temperature determination is one that makes use of Eq. (1) but relies on model atmospheres, rather than on a direct measure of the angular diameter. The infra red flux method (hereafter IRFM; Blackwell et al. 1990, and references therein) is especially adequate for determining the effective temperature of F, G and K stars. The IRFM was first introduced by Blackwell \& Shallis (1977) who proposed simultaneously determining the effective temperature and the angular diameter of a star. The basic idea is to use the monochromatic flux in the infrared since it is mainly dependent on the angular diameter but is approximately dependent only on the first power of $T_{\text {eff }}$, whereas the integrated flux strongly depends on the temperature (proportional to $T_{\text {eff }}^{4}$ ).

Popular indirect methods for deriving effective temperatures are the excitation equilibrium of Fe I lines (e.g. Santos et al. 2004, 2005) and on fitting Balmer lines (e.g. Fuhrmann et al. 1993, 1994; Barklem et al. 2002). Temperatures based on Fe I excitation equilibrium depend on the model assumptions, such as non-LTE effects, especially in metal-poor stars (see Thévenin \& Idiart 1999; Shchukina \& Trujillo Bueno 2001). 
Recently, Barklem (2007) has also raised concerns about possible non-LTE effects on the wings of Balmer lines. Both excitation equilibria (Asplund 2005) and Balmer lines (Ludwig et al. 2009, in prep.) are also sensitive to granulation effects. This makes such methods strongly model-dependent, which is an undesirable feature. However, temperatures derived from Balmer lines and Fe I excitation equilibria have the considerable advantage of being reddening independent.

One of the motivations of this work is to investigate the trend of $\mathrm{Li}$ abundances towards low metallicities $([\mathrm{Fe} / \mathrm{H}]<-2.5)$, using our own implementation of the IRFM. (Bonifacio et al. 2007) investigated the Spite plateau at the lowest metallicities (down to $[\mathrm{Fe} / \mathrm{H}]=-3.3$ ) and found marginal evidence that there could be an increased scatter or even a sharp drop in the $\mathrm{Li}$ abundance at these low metallicities. Determination of the baryonic density from the fluctuations in the cosmic microwave background (CMB) by the WMAP satellite (Spergel et al. 2003, 2007) implies a primordial $\mathrm{Li}$ abundance, which is at least a factor of 3-4 larger than observed on the Spite plateau, creating a conflict with the traditional interpretation of the plateau (Spite \& Spite 1982a,b). This discrepancy would be even greater if the drop in the Li abundance versus metallicity were to be confirmed (see Sbordone et al. 2008, in prep.).

One decade ago, Bonifacio \& Molaro (1997) determined Li abundances using the IRFM temperatures of Alonso et al. (1996a). They investigated the different $\mathrm{Li}$ abundance trends found with different temperature scales. In particular, the temperatures of Ryan et al. (1996), which are based on the IRFM implementation of Magain (1987), are cooler than the IRFM temperatures of Alonso et al. (1996a), provided $T_{\text {Alonso }}-T_{\text {Ryan }} \sim$ $+10 \mathrm{~K}$ at $[\mathrm{Fe} / \mathrm{H}] \sim-1.7$ and $\sim+130 \mathrm{~K}$ at $[\mathrm{Fe} / \mathrm{H}] \sim-3.3$. From this, Bonifacio \& Molaro (1997) concluded that the presence or absence of trends in lithium abundance with $T_{\text {eff }}$ is strongly dependent on the temperature scale adopted.

Meléndez \& Ramírez (2004) applied their own IRFM implementation (Ramírez \& Meléndez 2005a) to deriving the effective temperature and $\mathrm{Li}$ abundances for a sample of stars similar to that of Ryan et al. (1996). They find individual temperature differences of up to $400-500 \mathrm{~K}$ for the some stars with metallicity below -3.0 dex. More recently, Bonifacio et al. (2007) have compared the temperatures obtained from $\mathrm{H} \alpha$ profiles to other temperature indicators, among them those from the IRFM-based colour-temperature calibrations of (Ramírez \& Meléndez 2005a) and Alonso et al. (1996b). When a reddening based on the Schlegel et al. (1998) maps is adopted, from the $T_{\text {eff }}:(V-K)$ calibration of Alonso et al. (1996b), the mean difference $T_{(V-K)_{\mathrm{AAM} 96}}-T_{\mathrm{H} \alpha}$ is only $8 \mathrm{~K}$ with a standard deviation of $100 \mathrm{~K}$. However, if we use the $T_{\text {eff }}:(V-K)$ calibration of Ramírez \& Meléndez (2005b), this mean difference is $T_{(V-K)_{\mathrm{RM} 05}}-T_{\mathrm{H} \alpha}$ of $265 \mathrm{~K}$, with a standard deviation of $122 \mathrm{~K}$.

Ramírez \& Meléndez (2005a) add as calibrators a small sample of metal-poor stars mainly from Christlieb et al. (2004) and Cayrel et al. (2004), and a larger sample of metal-rich stars from Santos et al. (2004) to the original sample of Alonso et al. (1996a), and computed new $T_{\text {eff-colour calibrations. Since the }}$ majority of calibrators shared by the two samples, this large difference $(\sim 250 \mathrm{~K})$ between the calibrations of Ramírez \& Meléndez (2005a) and Alonso et al. (1996b) at low metallicity is a priori unexpected. One could argue that the models used by the two groups are not exactly the same, however they must be very similar (ATLAS 9 models with the same ODFs and microturbulent velocity). Since the IRFM is only weakly dependent on the models adopted, as shown by the results of Casagrande et al. (2006), who used both ATLAS and MARCS models, it seems
Table 1. Monochromatic Fluxes for Vega from the calibrated ATLAS 9 flux.

\begin{tabular}{lccc}
\hline \hline Band & $\begin{array}{c}\text { Wavelength } \\
\mathrm{nm}\end{array}$ & $\begin{array}{c}\text { Flux } \\
10^{-9} \\
\mathrm{erg} \mathrm{s}^{-1} \mathrm{~cm}^{-2} \mathrm{~nm}^{-1}\end{array}$ & Mag Vega \\
\hline$J$ & 1235 & 3.072 & 0.038 \\
$H$ & 1662 & 1.113 & 0.040 \\
$K$ & 2159 & 0.418 & 0.043 \\
\hline
\end{tabular}

unlikely that this difference is rooted in the different models. We suggest instead that this is because Ramírez \& Meléndez (2005a) use $2 \mathrm{MASS}^{1} J H K_{\mathrm{s}}$ magnitudes for the low metallicity calibrators; such magnitudes were then transformed into the TCS system to merge them with the homogeneous set of TCS photometry of Alonso et al. (1996a). The errors in the transformation between the 2MASS and the TCS systems are then added to the photometric error and may have undesired effects on the final calibration. We have no way to prove that this is indeed the case; however, to circumvent such problems in this paper, we propose a new implementation of the IRFM, including the stars from Alonso et al. (1996a, 1999a) and Ramírez \& Meléndez (2005a), but using the 2MASS photometry for all calibrators rather than a mixture of 2MASS and TCS. The 2MASS magnitudes are probably not as accurate as the careful TCS photometry of Alonso et al. (1996a), but the internal consistency of the 2MASS photometry is very high, about 1-2\% (Cutri et al. 2003).

\section{Implementation of the IRFM}

The IRFM (Blackwell et al. 1990) evaluates the quotient between the bolometric flux, $F_{\mathrm{bol}}$, and the monochromatic flux at a chosen infrared wavelength, $F\left(\lambda_{\mathrm{IR}}\right)$, both measured at the surface of the earth, as an indicator of the $T_{\text {eff }}$. This quotient is the so-called observational $R$-factor, $R_{\text {obs }}$. The theoretical counterpart derived from models, $R_{\text {theo }}$, is obtained as the quotient between the integrated flux, $\sigma T_{\text {eff }}^{4}$, and the monochromatic flux at $\lambda_{\mathrm{IR}}, F_{\bmod }\left(\lambda_{\mathrm{IR}}\right)$, at the surface of the star. Thus the basic equation of the IRFM is

$$
\begin{aligned}
R_{\mathrm{obs}} & =\frac{F_{\mathrm{bol}}}{F\left(\lambda_{\mathrm{IR}}\right)}=\frac{\sigma T_{\mathrm{eff}}^{4}}{F_{\mathrm{mod}}\left(\lambda_{\mathrm{IR}}, T_{\mathrm{eff}},[\mathrm{Fe} / \mathrm{H}], \log g\right)} \\
& =R_{\mathrm{theo}}\left(\lambda_{\mathrm{IR}}, T_{\mathrm{eff}},[\mathrm{Fe} / \mathrm{H}], \log g\right)
\end{aligned}
$$

where the dependence of models on metallicity, surface gravity, and $\lambda_{\mathrm{IR}}$ is explicitly taken into account. The monochromatic fluxes are obtained by applying the relation

$$
F\left(\lambda_{\mathrm{IR}}\right)=q\left(\lambda_{\mathrm{IR}}, T_{\mathrm{eff}},[\mathrm{Fe} / \mathrm{H}], \log g\right)\left[F_{\mathrm{cal}}\left(\lambda_{\mathrm{IR}}\right) 10^{-0.4\left(m_{\star}-m_{\mathrm{cal}}\right)}\right]
$$

where $m_{\star}$ is the magnitude of the target star, and $m_{\text {cal }}$ and $F_{\text {cal }}$ are, respectively, the magnitudes and the absolute monochromatic fluxes of the calibrator star (see Table 1 and Sect. 5). The $q$-factor, usually $\sim 1$, is a dimensionless factor that corrects the effect of the different curvature of the flux distribution across the filter window (see Alonso et al. 1994, 1996a, 1999a, for more details). We have used the definition of Alonso et al. (1996a) for the computations of the $q$-factors (see Sect. 5).

\footnotetext{
1 The 2MASS catalogue can be accessed at http://www . ipac. caltech. edu/2mass/.
} 
By merging the previous two equations we can separate the observational and model inputs as

$$
\begin{aligned}
\frac{F_{\text {bol }}}{F_{\text {cal }}\left(\lambda_{\mathrm{IR}}\right) 10^{-0.4\left(m_{\star}-m_{\text {cal }}\right)}}= & q\left(\lambda_{\mathrm{IR}}, T_{\mathrm{eff}},[\mathrm{Fe} / \mathrm{H}], \log g\right) \\
& \times R_{\text {theo }}\left(\lambda_{\mathrm{IR}}, T_{\mathrm{eff}},[\mathrm{Fe} / \mathrm{H}], \log g\right) .
\end{aligned}
$$

The synthetic magnitudes, the $q$ - and $R$-factors, necessary for implementing of the IRFM were computed from the ATLAS theoretical fluxes of Castelli \& Kurucz $(2003)^{2}$ using the 2MASS $J H K_{\mathrm{s}}$ filters. We used the fluxes in the ranges $3500 \mathrm{~K}<T_{\text {eff }}<$ $7500 \mathrm{~K}, 0.0<\log g<5.0$, and $-4<[\mathrm{Fe} / \mathrm{H}]<+0.5$, and for metal-poor models with $[\mathrm{Fe} / \mathrm{H}] \leq-0.5$, we used the fluxes computed from the $\alpha$-enhanced models. We derived a new calibration of the bolometric flux in the 2MASS photometric system (see Sect. 6).

\section{Online data available at the CDS}

Several tables are available at the $\mathrm{CDS}^{3}$. We provide eight tables containing the $q$ - and $R$-factors computed as indicated in Sects. 2 and 5 for metallicities in the range [-4,0.5], temperatures in the range [3500, 50 000], and gravities in the range [0,5]. Within these tables, we also put the theoretical colour $V-K$ and magnitudes $J H K \mathrm{~s}$ in the 2MASS system for each atmospheric model. These theoretical colour and magnitudes, which are not used in this work, were normalised to Vega assuming $V=J=H=K \mathrm{~s}=0$. If the user wants to use a different zero point for Vega, it is trivial to add it to our theoretical magnitudes. In addition, eight tables containing the $A$-factors and $B X-B$ coefficients for the same set of models are also available at the CDS, needed for the bolometric flux calibration (see Sect. 6). At the CDS, we also provide two tables, with 555 dwarf stars and with 264 giant stars, containing the photometric data and reddenings used in this paper, stellar parameters and metallicity, bolometric fluxes and IRFM temperatures for all the stars in our samples (see Sects. 4.1 and 4.2).

\section{Sample, observational data, and stellar parameters}

\subsection{Photometric data}

Our sample includes almost all the stars in Alonso et al. (1996a, 1999a) with available photometric data in the final release of the 2MASS catalogue (Skrutskie et al. 2006) and with photometric accuracy $\lesssim 0.3 \mathrm{mag}$, for temperature determinations. We adopted this rather high tolerance because giant stars of Alonso et al. (1999a) are relatively bright and usually the 2MASS photometric accuracy is very low for these stars. However, to improve the precision of the bolometric flux and $T_{\text {eff }}$ :colour $-[\mathrm{Fe} / \mathrm{H}]$ calibrations significantly, we decided to further lower the accuracy limit down to $\lesssim 0.1 \mathrm{mag}$ (see Sects. 6 and 9). Therefore, stars with 2MASS photometric errors $>0.1$ mag were only used for the purpose of deriving effective temperatures and they are provided as online data at the CDS.

We adopted the same $U B V(R I)_{\mathrm{C}}$ photometric data as used by Ramírez \& Meléndez (2005a,b), which were kindly provided by Ramírez \& Meléndez (private communication). These data were extracted from the General Catalogue of Photometric Data (Mermilliod et al. 1997, GCPD). For those stars of the

\footnotetext{
2 http://wwwuser.oats.inaf.it/castelli/grids.html

${ }^{3}$ http://cdsweb.u-strasbg.fr/
}

Alonso et al. (1996a, 1999a) samples without $V$ data in the GCPD these magnitudes were obtained from $\mathrm{Simbad}^{4}$, and were later used to derive bolometric fluxes and IRFM temperatures.

\subsection{Metallicity and surface gravity}

For our sample of stars from (Alonso et al. 1996a, 1999a), we adopted the surface gravities and metallicities provided by Ramírez \& Meléndez (private communication) which mostly use the mean values of those reported in Cayrel de Strobel et al. (2001).

We completed our sample of dwarfs and subgiants with the metal-rich and metal-poor stars already included in the sample of Ramírez \& Meléndez (2005a). The metal-rich sample mostly contains planet-host stars and the comparison sample from Santos et al. (2004), but we also added to our sample the stars with $[\mathrm{Fe} / \mathrm{H}]>-2$ from Casagrande et al. (2006). We completed the sample with extremely metal-poor dwarfs from Bonifacio et al. (2007), Christlieb et al. (2004), and Barklem et al. (2005). For these stars, we adopted the same surface gravity and metallicity as published in the above papers.

Our sample of giants contains the stars in Alonso et al. (1999a), plus the metal-poor stars from Ramírez \& Meléndez (2005a). This includes stars from the "First Stars" project (Cayrel et al. 2004; Spite et al. 2005), and we adopted the surface gravity and metallicity for these stars as provided in these papers.

The errors on surface gravity and metallicity for all dwarf, subgiant, and giant stars were assumed to be $\Delta \log g=0.5 \mathrm{dex}$ and $\Delta[\mathrm{Fe} / \mathrm{H}]=0.1$. The average systematic errors due to a different metallicity (by $+0.1 \mathrm{dex}$ ) and a different surface gravity (by $+0.5 \mathrm{dex})$ are $13 \mathrm{~K}$ and $11 \mathrm{~K}$, respectively, for dwarfs, and $11 \mathrm{~K}$ and $28 \mathrm{~K}$ for giants. These errors were estimated by quadratically adding the errors on effective temperature from each band and calculating the average over all stars in both samples.

\subsection{Reddening corrections}

The extinction in each photometric band, $A_{i}$, as determined using the relation $A_{i}=R_{i} E(B-V)$, where $R_{i}$ is given by the coefficients provided in (McCall 2004). Reddening corrections, $E(B-V)$, were adopted from Ramírez \& Meléndez (private communication). For the metal-rich stars of Casagrande et al. (2006) and the extremely metal-poor dwarfs of Bonifacio et al. (2007) and Christlieb et al. (2004), reddening corrections were derived from the maps of Schlegel et al. (1998). The $E(B-V)$ from the maps is appropriate for objects outside the dust layer, which is confined to the Galactic disc. For objects which are within the dust layer the map estimate should be corrected by a factor $[1-\exp (-|d \sin b| / h)]$, where $d$ is the distance of the star, $b$ its galactic latitude and $h$ the scale height of the dust layer (see, e.g. Bonifacio et al. 2000b). For this purpose we used the parallaxes provided by Simbad (which come mainly from the Hipparcos catalogue Perryman et al. 1997) and assumed a scale height of the dust layer of $125 \mathrm{pc}$. Bonifacio et al. (2000a) note that, when the maps of Schlegel et al. (1998) provide reddenings larger than $0.1 \mathrm{mag}$, they overestimate the reddening with respect to other indicators, and proposed a simple formula for correcting the reddening from the maps. We make use of formula (1) of Bonifacio et al. (2000a) to correct the reddenings derived from the maps of Schlegel et al. (1998).

\footnotetext{
${ }^{4}$ http://simbad.u-strasbg.fr/simbad/.
} 


\section{Photometric zero points and absolute flux calibrations for use with the IRFM}

Equation (4) is what needs to be implemented practically to derive IRFM temperatures. The quantities on the lefthand side are observed quantities while those on the righthand side are theoretical quantities. One is immediately faced with a series of choices

1. the magnitude of the standard star $\left(m_{\mathrm{cal}}\right)$;

2. the monochromatic flux of the standard $\operatorname{star}\left(F_{\mathrm{cal}}\right)$;

3 . the zero point for $q$;

4. the zero point for $R_{\text {theo }}$.

These choices are only apparently trivial. The 2MASS magnitudes have been carefully calibrated in absolute fluxes by Cohen et al. (2003); however, the standard star to which the whole system is tied, Vega, has not been observed by 2MASS with sufficient accuracy due to its high brightness. A possible solution is to assume that the 2MASS magnitudes of Vega are given by the zero points of Cohen et al., with changed sign, as in Casagrande et al. (2006). Another complication is the zero point of the theoretical quantities. It is obvious from the definition of $q$ that its value is 1 for the standard star; however, what are the correct effective temperature, metallicity, and surface gravity of the standard star? The Cohen et al. calibration relies on an ATLAS theoretical spectrum computed by Kurucz with the "OLD" opacity distribution functions assuming $T_{\mathrm{eff}}=9400, \log g=3.9$, a metallicity of -0.5 , and a microturbulent velocity of $0 \mathrm{~km} \mathrm{~s}^{-1}$. Such a spectrum is not available in tabular form, we could indeed recompute it, however using such a spectrum to zero our theoretical quantities would mean using a spectrum that is computed from a model inconsistent with the rest of the theoretical grid. Furthermore, as we shall see in Sect. 6, we will also need the absolute fluxes in other bands to derive a calibration for the bolometric flux. The natural choice is to use the corresponding theoretical magnitudes of Bessell et al. (1998) transformed into the 2MASS system. These magnitudes rely on the model for Vega, proposed by Castelli \& Kurucz (1994), consistent with the grid of Castelli \& Kurucz (2003) that we are using. A possible solution is to follow what was done by Casagrande et al. (2006), who in fact used two different calibrations for optical and IR magnitudes. Inspection of Eq. (4) suggests another solution: use the same spectrum of the standard star to calibrate all bands. In this way any error in the calibration will cancel out when computing the flux ratio on the lefthand side of Eq. (4). However, to have a good absolute calibration, one also needs accurate observed or derived 2MASS magnitudes of the standard star Vega, which is quite difficult to obtain. We decided to adopt as 2MASS magnitudes of Vega those provided by McCall (2004, see Sect. 6). This theoretical spectrum should also be used to define the zero point of $q$ and $R_{\text {theo }}$ for the standard star, in order to have a fully self-consistent IRFM.

Throughout this work we adopt the theoretical flux of Vega of Castelli \& Kurucz (1994) ${ }^{5}$, which has been calibrated to absolute flux, at Earth, using the value recommended by Hayes $\left(1985,3.44 \times 10^{-8} \mathrm{erg} \mathrm{s}^{-1} \mathrm{~cm}^{-2} \mathrm{~nm}^{-1}\right)$. This spectrum is used to define the zero point of the $q$ factor and the monochromatic fluxes, at the isophotal wavelengths of the 2MASS filters, listed in Table 1 are used in our implementation of Eq. (4). As noted by Casagrande et al. (2006), such a calibrated spectrum differs to the one used by Cohen et al. (1992) and adopted by Cohen et al. (2003) to define the absolute flux calibration of the 2MASS

\footnotetext{
5 http://wwwuser.oats.inaf.it/castelli/vega/ fm05t9550g395k2odfnew.dat
}

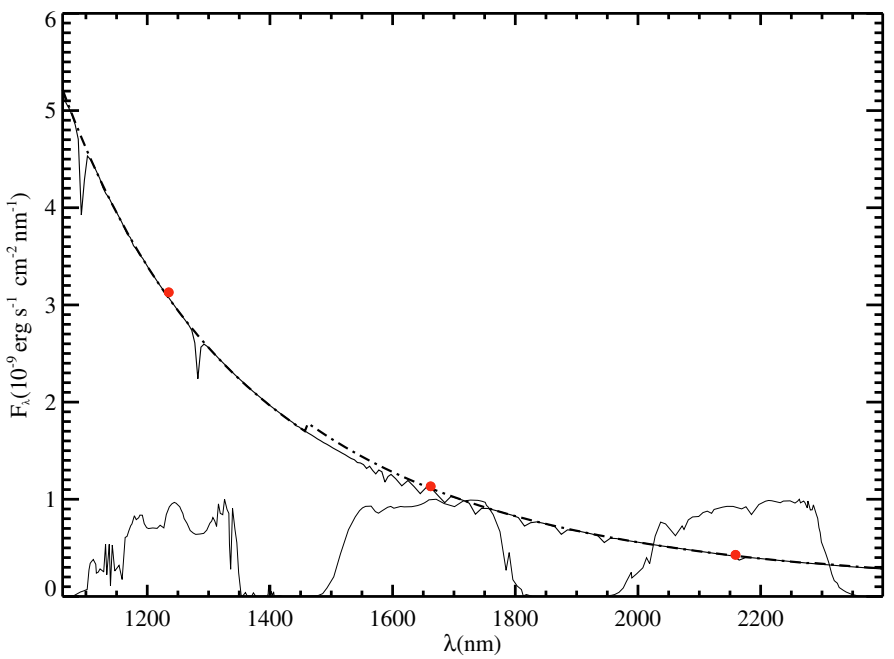

Fig. 1. Spectral energy distribution of the ATLAS model of Vega $\left(T_{\mathrm{eff}}=\right.$ $9550 \mathrm{~K}, \log g=3.95,[\mathrm{Fe} / \mathrm{H}]=-0.5$ and $v_{\text {micro }}=2 \mathrm{~km} \mathrm{~s}^{-1}$ ) in the infrared. The solid and dashed-dotted lines represent the intensity and continuum flux, respectively. The filled circles are the monochromatic fluxes adopted by Cohen et al. (2003). We also show the transmission functions of the $\mathrm{JHK}_{\mathrm{s}}$ 2MASS filters.

magnitudes. In Fig. 1 we display the calibrated spectrum of Vega in comparison with the adopted monochromatic fluxes of Cohen et al. (2003). The difference is small when comparing them with the continuum flux of our ATLAS 9 model of Vega at the same infrared wavelenghts. We stress that, for the purpose of consistent IRFM temperatures, we are not all that interested in having accurate monochromatic fluxes, but instead accurate ratios of bolometric fluxes to monochromatic fluxes. However, it should be noted that the adopted observed magnitudes for Vega are perhaps the source of uncertainty in a given temperature scale based on the IRFM.

We adopted an error of 1 per cent on the monochromatic flux of each band for the determination of effective temperatures. The average systematic errors due to this uncertainty are $44 \mathrm{~K}$ and $46 \mathrm{~K}$ for dwarfs and giants, respectively. These errors were estimated by adding the errors quadratically on temperature from each band and calculating the average over all stars in both samples.

\section{Bolometric fluxes}

One of the fundamental observational quantities for applying the IRFM is the bolometric flux. The bolometric flux is not readily available for any given star, but Blackwell \& Petford (1991) suggested that one could use a relation of the type $F_{\text {bol }}=10^{-0.4 m} \phi(X,[\mathrm{Fe} / \mathrm{H}])$, where $m$ is a suitable broad band colour and $X$ a colour index. Such a calibration has been derived by Alonso et al. (1995) using the $K$ magnitude and the $V-K$ colour (Johnson system); Casagrande et al. (2006) have derived several similar calibrations for different choices of $m$ and the colour index. In an initial attempt we tried to use the Alonso et al. (1995) calibration for this purpose, which provided satisfactory results; however, the referees have correctly pointed out that, in doing so, we were forced to transform our $\left(V-K_{\mathrm{S}}\right)$ colour into Johnson's system, thus losing the internal consistency of the 2MASS system. Furthermore, we had to apply the Alonso et al. (1995) calibration outside its formal range of applicability, for very metal-poor stars. The calibration of Alonso et al. (1995) only had two stars at $[\mathrm{Fe} / \mathrm{H}]=-3.2$ and -2.9 and the rest with 
$[\mathrm{Fe} / \mathrm{H}]>-2.6$. A similar extrapolation problem would apply if we had used any of the calibrations derived by Casagrande et al. (2006), which were derived for stars with $[\mathrm{Fe} / \mathrm{H}]>-1.9$. We therefore decided to derive a new calibration that makes use of the $K_{\mathrm{s}}$ magnitude and the 2MASS-based $\left(V-K_{\mathrm{s}}\right)$ colour and covers the metallicity range appropriate to our sample of stars.

We adopt an approach similar to that of Alonso et al. (1995) and Casagrande et al. (2006), with a slight difference. The above authors use a set of effective wavelengths and monochromatic fluxes for Vega in order to define the integrated flux within each broad band from the photometry and the magnitudes of Vega. From the definition of magnitude follows

$F_{*}=F_{\text {Vega }} 10^{-0.4\left(m-m_{\text {Vega }}\right)}$

where $m$ is any photometric band. Provided then that the integrated flux of Vega in any given band is known, the integrated flux for the target star may be simply derived from its measured magnitude and the magnitude of Vega. In Table 3 we provide our adopted integrated magnitudes for Vega for the bands we are interested in, and $J H K_{\mathrm{s}}$ refer to the 2MASS colours. These integrated magnitudes were derived by integrating the filter response functions of Bessell (1990) for the optical bands and Cohen et al. (2003) for the 2MASS bands, over the theoretical flux of Vega. Consistently, the magnitudes for Vega were taken from McCall (2004), which gives the model magnitudes of Bessell et al. (1998) for the optical bands. This author calculates the 2MASS magnitudes of Vega using the IR absolute monochromatic fluxes from Cohen et al. (2003), which are in fact quite similar to our adopted absolute monochromatic fluxes from the calibrated model of Vega (see Fig. 1). Initially we were going to adopt the magnitudes of Vega equal to zero in the 2MASS bands, but when we derived the IRFM temperatures, our scale of temperatures was $\sim 120 \mathrm{~K}$ hotter than that of Alonso et al. (1996a), which we think is the best implementation of the IRFM available in the literature due to its internal consistency in the whole range of metallicities from -3.0 to 0.5 . By adopting the magnitudes of Vega given by McCall (2004), this difference is reduced to $\sim 60 \mathrm{~K}$, which we consider more appropriate (see Sect. 8.1). In addition, the remarks on zero points of Sect. 5 also apply here. This choice guarantees that any error in the absolute calibration of bolometric fluxes and monchromatic fluxes will cancel out in Eq. (4).

From Eq. (5) and the data in Table 3 for any star for which photometry in several bands is available, one may computed the total flux measured at Earth in the given bands. From this value, the bolometric flux can be obtained by using model data.

In practice the stars we decided to use to derive the bolometric flux calibrations fall into one of four groups:

1. stars with full $U B V(R I)_{\mathrm{C}} J H K_{\mathrm{s}}$ data;

2. stars with $U B V J H K_{\mathrm{s}}$ data;

3. stars with $B V J H K_{\mathrm{S}}$ data;

4. stars with $B V(R I)_{\mathrm{C}} J H K_{\mathrm{s}}$ data.

The information is more complete for the stars of group 1) and 4); however, we decided to include the also the stars of groups 2) and 3 ) in our calibration effort, since this allows us to include a relevant number of stars at extremely low metallicity.

For each group one may compute

$F_{\text {obs }}=\sum_{i} \int F(\lambda) T_{i}(\lambda) \mathrm{d} \lambda$

where the sum is extended to all the available bands and $T_{i}(\lambda)$ is the response function of the ith band, the integrals must be considered extended from 0 to infinity, formally, although in practise the response function of each filter vanishes outside a finite interval and numerically one stops integrating outside this interval. One can then use the models to compute the correction

$F_{\text {bol }}=A F_{\text {obs }}$

where $A$ is a function of $T_{\text {eff }}, \log g$ and $[\mathrm{Fe} / \mathrm{H}]$. Obviously a different $A$ has to be computed for any given set of available bands. The $A$ factors for the different band combinations we have used are given in machine readable form at the CDS (see Sect. 3) with different subscripts 1 to 4 corresponding to the different band combinations. This is again slightly different from what was done by Alonso et al. (1995) or Casagrande et al. (2006), who used the monochromatic fluxes at the effective wavelengths of each band to approximate the spectral energy distribution of the star and integrated this approximate energy distribution over the whole interval. We only make use of integrated fluxes, which are related to the observed magnitudes through Eq. (5) and of the fluxes and magnitudes of Vega given in Table 3.

While the 2MASS magnitudes are provided by the catalogue, it is customary for the optical bands to provide the $V$ magnitude and the colours $U-B, B-V, V-R, V-I$. While for the bands $B$ to $I$ it is straightforward to obtain the magnitude (e.g. $R=V-(V-R)$ ), some caution must be exerted for the $U$ band, for which the atmospheric extinction is strongly varying across the band and establishes the UV cut-off. In fact, this band has proved to be the most difficult to standardize. Bessell (1990) provides a response curve $U X$ in which the atmospheric extinction is folded in and gives the curves $B X$ and $B$ for the $B$ band. The former is to be used to compute the synthetic photometry of the $(U-B)$ colour, while the latter is to be used to compute the synthetic $(B-V)$ colour. For the stars to be used in our bolometric flux calibration we define the $U$ magnitude

$U=(U-B)+V+(B-V)+(B X-B)$

where $V,(U-B)$, and $(B-V)$ are the observed magnitude and colours of the star, and $(B X-B)$ is derived from the theoretical models with the requirement that it must be equal to zero for Vega. The colours $(B X-B)$ are given in the online data at the CDS (see Sect. 3).

The bolometric flux of each star was determined as in Alonso et al. (1995). We first determined the fluxes of each band by applying Eq. (5) from the observed magnitudes of the star. Then we derived the bolometric flux, $F_{\text {bol }}$, using the Eq. (7). Thus the temperature $T_{\text {eff }}$ was then determined using the IRFM that combines $F_{\text {bol }}$ and the monochromatic fluxes at IR wavelengths. This new value for the effective temperature may re-enter in Eq. (7) to derive a new value for $F_{\text {bol }}$, and so on. This iterative procedure converges quickly towards a final $F_{\text {bol }}$. In each iteration, the factors $A$ and $B X-B$ were determined using a trilinear interpolation within the grid for the corresponding $T_{\text {eff }}, \log g$, and $[\mathrm{Fe} / \mathrm{H}]$ of the star. We considered the errors on the IRFM $T_{\text {eff }}$ due to uncertainties on the adopted absolute calibration of the 2MASS photometric system, and the errors on the magnitudes $J H K_{\mathrm{s}} \log g$, and $[\mathrm{Fe} / \mathrm{H}]$.

We derived relations between bolometric fluxes and colours, taking also the effects of metallicity into account. We adopted the same fitting formula as adopted by Casagrande et al. (2006)

$$
\begin{aligned}
\phi(X,[\mathrm{Fe} / \mathrm{H}])= & a_{0}+a_{1} X+a_{2} X^{2}+a_{3} X^{3} \\
& +a_{4} X[\mathrm{Fe} / \mathrm{H}]+a_{5}[\mathrm{Fe} / \mathrm{H}]+a_{6}[\mathrm{Fe} / \mathrm{H}]^{2}
\end{aligned}
$$

where the $\phi$ is derived as $\phi(V-m,[\mathrm{Fe} / \mathrm{H}])=F_{\text {bol }} / 10^{-0.4 m}, X=$ $V-m$ represents the $J, H, K_{\mathrm{s}}$ magnitudes, and $a_{i}(i=0, \ldots, 6)$ are the coefficients of the fit. We iterate the fitting procedure by 

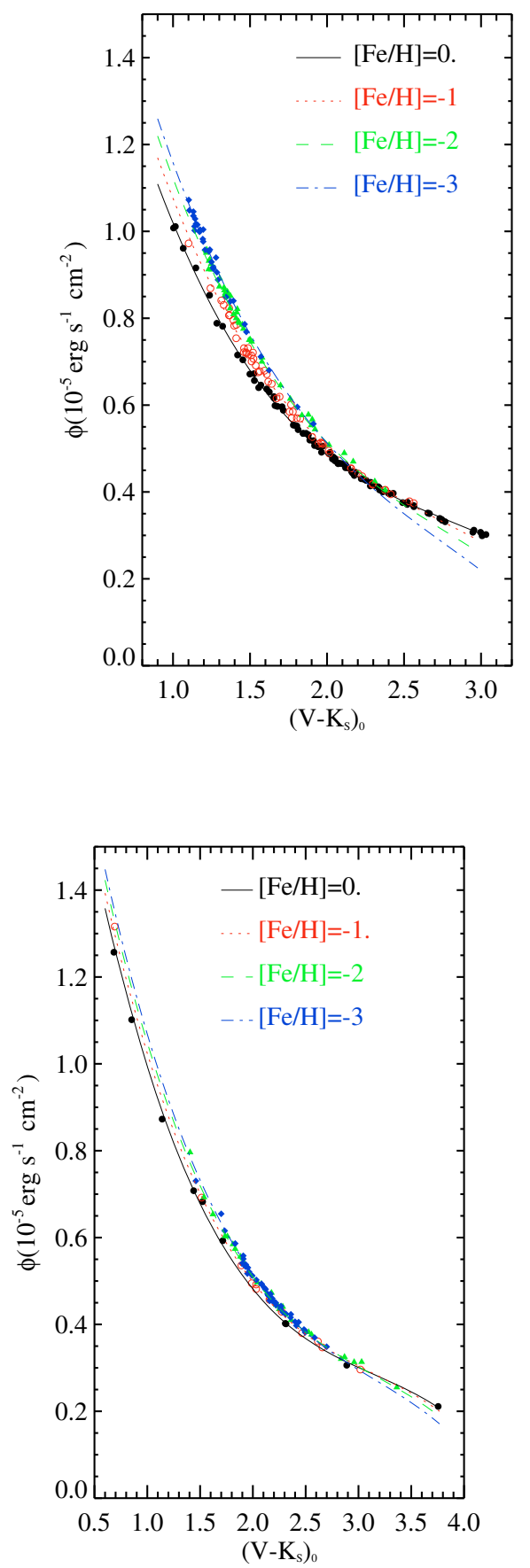
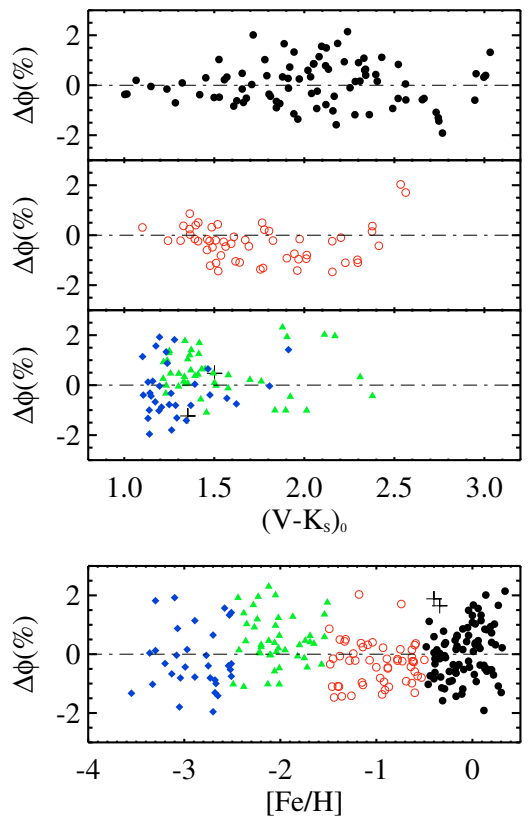

Fig. 2. Left: empirical calibration $\phi:\left(V-K_{\mathrm{s}}\right)-$ $[\mathrm{Fe} / \mathrm{H}]$ for dwarfs in the metallicity bins $-0.5<$ $[\mathrm{Fe} / \mathrm{H}] \leq 0.5$ (filled circles), $-1.5<[\mathrm{Fe} / \mathrm{H}] \leq$ -0.5 (open circles), $-2.5<[\mathrm{Fe} / \mathrm{H}] \leq-1.5$ (triangles), and $[\mathrm{Fe} / \mathrm{H}] \leq-2.5$ (diamonds). The lines correspond to our calibration for $[\mathrm{Fe} / \mathrm{H}]=$ 0 (solid line), -1.0 (dotted line), -2.0 (dashed line), -3.0 (dotted-dashed line). Right: residuals of the fit $\left(\Delta \phi=\left(\phi_{\text {cal }}-\phi_{\text {IRFM }}\right) / \phi_{\text {IRFM }}\right)$ as a function of $\left(V-K_{\mathrm{s}}\right)$ and $[\mathrm{Fe} / \mathrm{H}]$.
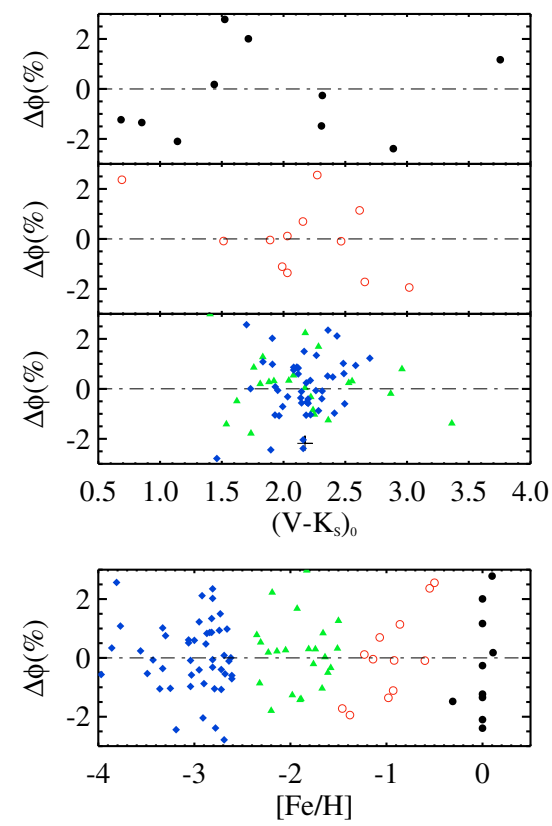

Fig. 3. The same as Fig. 2, but for giants.

discarding the points more than $2.5 \sigma$ from the mean fit. We also tried other fitting formulae such as that of Alonso et al. (1995), but they led to similar results. This has been extensively tested by Casagrande et al. (2006), who also give fits using optical bands.

For dwarf stars, we selected all the stars of group 1) from the sample of Alonso et al. (1996a) and Ramírez \& Meléndez (2005a) with uncertainties in the $J H K_{\mathrm{s}}$ magnitudes less than 0.1 at all metallicities. We added the stars of group 4) from the sample of Casagrande et al. (2006) with the same restrictions and we completed the sample with stars of groups 2) and 3) with $[\mathrm{Fe} / \mathrm{H}]<-2$. We only added metal-poor stars to give more weight to the metal-rich stars of groups 1) and 4); otherwise, we would end up with including all dwarf stars and the fit would be dominated by the greater number of stars of group 3).

For giant stars, we applied the same restrictions but the number of stars with Alonso et al. (1996a) and Ramírez \& Meléndez (2005a) of groups 1) and 4) was very small (only 10 stars in group 1) and 26 in group 4) with uncertainties in the $J H K_{\mathrm{s}}$ magnitudes less than 0.1 ), so we decided to include all the stars of groups 2) and 3).

In Figs. 2 and 3 we display the polynomial fits that represent the empirical calibrations $\phi$ versus colours and metallicity. The coefficients of these calibrations are given in Table 2, together with the remaining number of stars after the $2.5 \sigma$ clipping and the rms of the fit, $\sigma_{\phi}$. These calibrations show similar behaviours 
Table 2. Coefficients and range of applicability of the $\phi:(V-m)-[\mathrm{Fe} / \mathrm{H}]$ calibrations.

\begin{tabular}{|c|c|c|c|c|c|c|c|c|c|c|c|}
\hline Colour & Colour range & {$[\mathrm{Fe} / \mathrm{H}]$ range } & $a_{0}^{a}$ & $a_{1}$ & $a_{2}$ & $a_{3}$ & $a_{4}$ & $a_{5}$ & $a_{6}$ & $N^{b}$ & $\overline{\sigma_{\phi}(\%)^{c}}$ \\
\hline \multicolumn{12}{|c|}{ Dwarf stars } \\
\hline$V-J$ & {$[0.8,2.4]$} & {$[-3.5,0.3]$} & 2.4945 & -2.2635 & 0.9615 & -0.1509 & 0.0657 & -0.1365 & -0.0074 & 219 & 0.9 \\
\hline$V-H$ & {$[0.9,3.0]$} & {$[-3.5,0.3]$} & 2.3681 & -1.9055 & 0.6415 & -0.0773 & 0.0418 & -0.1028 & -0.0053 & 216 & 0.9 \\
\hline$V-K_{\mathrm{s}}$ & {$[1.0,3.0]$} & {$[-3.5,0.3]$} & 2.3522 & -1.8817 & 0.6229 & -0.0745 & 0.0371 & -0.0990 & -0.0052 & 216 & 0.9 \\
\hline \multicolumn{12}{|c|}{ Giant stars } \\
\hline$V-J$ & {$[0.5,2.7]$} & {$[-4.0,0.1]$} & 2.2282 & -1.7818 & 0.6809 & -0.0923 & 0.0302 & -0.0696 & -0.0031 & 97 & 1.3 \\
\hline$V-H$ & {$[0.6,3.4]$} & {$[-4.0,0.1]$} & 2.1522 & -1.5792 & 0.4821 & -0.0523 & 0.0182 & 0.0502 & -0.0019 & 91 & 1.3 \\
\hline$V-K_{\mathrm{s}}$ & {$[0.7,3.8]$} & {$[-4.0,0.1]$} & 2.1304 & -1.5438 & 0.4562 & -0.0483 & 0.0132 & 0.0456 & -0.0026 & 95 & 1.4 \\
\hline
\end{tabular}

${ }^{a}$ The coefficients of the calibrations $a_{i}$ are given in units of $10^{-5} \mathrm{erg} \mathrm{cm}^{-2} \mathrm{~s}^{-1}$.

${ }^{b} N$ is the remaining number of stars after several iterations (usually less than 20 ) of the $2.5 \sigma$ clipping.

${ }^{c} \sigma_{\phi}$, given in per cent, is the standard deviation of the final calibrations.

Table 3. Adopted absolute integrated fluxes and magnitudes for Vega.

\begin{tabular}{ccc}
\hline \hline Band & $\begin{array}{c}\text { Flux } \\
10^{-5} \\
\mathrm{erg} \mathrm{s}^{-1} \mathrm{~cm}^{-2}\end{array}$ & mag(Vega) \\
\hline$U$ & 0.267 & 0.024 \\
$B$ & 0.607 & 0.028 \\
$V$ & 0.321 & 0.030 \\
$R$ & 0.341 & 0.037 \\
$I$ & 0.167 & 0.033 \\
$J$ & 0.050 & 0.038 \\
$H$ & 0.028 & 0.040 \\
$K$ & 0.011 & 0.043 \\
\hline
\end{tabular}

to those of Alonso et al. (1995) and Alonso et al. (1999a), at least in the metallicity range from -3 to 0 .

\section{IRFM temperatures and angular diameters}

To determine effective temperatures we need to apply Eq. (4). The bolometric fluxes are estimated using the empirical calibration $F_{\text {bol,cal }}=10^{-0.4 K_{\mathrm{s}}} \phi\left(V-K_{\mathrm{s}},[\mathrm{Fe} / \mathrm{H}]\right)$ given in Table 2 and the 2MASS $K_{\mathrm{s}}$ and Johnson $V$ magnitudes. The $q$ - and $R$-factors are determined from an initial guess of the temperature of the star, $T_{\text {eff } 0}$, by trilinear interpolation in the grid, using the surface gravity and metallicity of the star. Then, we determine a new value for the temperature by comparing the theoretical bolometric flux, $F_{\text {bol,theo }}$, derived from the previous determination of $T_{\text {eff }}$ and the bolometric flux, $F_{\text {bol,cal }}$, using the expression: $T_{\text {eff, new }}=T_{\text {eff,old }}\left[F_{\text {bol,cal }} / F_{\text {Bol,theo }}\right]^{1 / 4}$. We again derive the $q$ - and $R$-factors for $T_{\text {eff, new }}$ and repeat this process iteratively until $\left|T_{\text {eff,new }}-T_{\text {eff,old }}\right| \leq 0.1 \mathrm{~K}$.

The final temperature of the star is determined as the average of the three temperatures extracted from each of 2MASS filters weighted with the inverse of their individual errors (see Alonso et al. 1996a). The error on the weighted mean is computed as $\Delta T_{\text {eff }}=N / \sum\left(\Delta T_{i}\right)^{-1}$ where $\Delta T_{i}$ are the errors of the temperatures from the individual filters $\left(i=J, H, K_{\mathrm{s}}\right)$ and $N=3$ is the number of available temperatures. These errors $\Delta T_{i}$ account for the photometric errors of the observed $J H K_{\mathrm{s}}$ and $V$ magnitudes, the error on the adopted absolute calibration for the 2MASS photometric system, and the uncertainties on surface gravity and metallicity. To estimate $\Delta T_{i}$, we just add all the individual errors of the $i$ band quadratically.

The angular diameters have been calculated from Eq. (1) with the derived IRFM temperatures and bolometric fluxes. Their errors were estimated by propagating a mean error of $1.3 \%$ in the bolometric fluxes and the errors on $T_{\text {eff }}$.
Table 4. Comparison with other temperature scales.

\begin{tabular}{lcrrr}
\hline \hline Sample & {$[\mathrm{Fe} / \mathrm{H}]$ range } & $\Delta T_{\text {eff }}$ & $\sigma_{T_{\text {eff }}}$ & $N^{a}$ \\
\hline & Dwarf Stars & & & \\
Alonso et al. (1996a) & {$[-3.5,+0.3]$} & +64 & 104 & 332 \\
Alonso et al. (1996a) & {$[-3.5,-2.5]$} & +61 & 91 & 18 \\
Alonso et al. (1996a) & {$[-0.5,+0.3]$} & +32 & 130 & 122 \\
Ramírez \& Meléndez (2005a) & {$[-4.0,+0.3]$} & +33 & 98 & 84 \\
Ramírez \& Meléndez (2005a) & {$[-4.0,-2.5]$} & -87 & 194 & 12 \\
Ramírez \& Meléndez (2005a) & {$[-0.5,+0.3]$} & +45 & 91 & 69 \\
Casagrande et al. (2006) & {$[-1.9,+0.4]$} & -12 & 56 & 101 \\
Casagrande et al. (2006) & {$[-1.9,+0.4]$} & -41 & 50 & 101 \\
Santos et al. (2004) & {$[-0.7,+0.5]$} & +11 & 120 & 133 \\
Santos et al. (2004) & {$[-0.7,+0.5]$} & -13 & 129 & 133 \\
Bonifacio et al. (2007) & {$[-3.6,-2.4]$} & +165 & 79 & 16 \\
Barklem et al. (2002) & {$[-2.5,+0.1]$} & +77 & 133 & 23 \\
Barklem et al. (2002) & {$[-0.5,-0.1]$} & +51 & 129 & 16 \\
Christlieb et al. (2004) & {$[-3.1,-1.6]$} & +177 & 80 & 8 \\
Baines et al. (2008) & {$[-0.4,0.5]$} & -32 & 163 & 22 \\
\hline & Giant stars & & & \\
Alonso et al. (1999a) & {$[-3.0,+0.5]$} & +54 & 131 & 202 \\
Alonso et al. (1999a) & {$[-3.0,-2.5]$} & +76 & 120 & 10 \\
Alonso et al. (1999a) & {$[-0.5,+0.5]$} & +43 & 144 & 116 \\
Ramírez \& Meléndez (2005a) $^{b}[-4.0,+0.3]$ & +63 & 57 & 25 \\
Ramírez \& Meléndez (2005a) $^{b}[-4.0,-2.5]$ & +61 & 62 & 18 \\
Ramírez \& Meléndez (2005a) & $+0.2^{d}$ & +116 & - & 1 \\
Cayrel et al. (2004) & {$[-4.0,-2.0]$} & +115 & 76 & 34 \\
Christlieb et al. (2004) & {$[-3.4,-2.6]$} & +128 & 71 & 22 \\
Baines et al. (2008) & 0 & -67 & 139 & 6 \\
\hline
\end{tabular}

${ }^{a}$ The number of stars.

${ }^{b}$ If we consider all reddening corrections equal to zero.

${ }^{c} \Delta T_{\mathrm{eff}}=T_{\mathrm{eff}}^{\mathrm{IRFM}}-T_{\mathrm{eff}}^{\mathrm{dir}}$, where $T_{\mathrm{eff}}^{\mathrm{dir}}$ is a direct determination of $T_{\mathrm{eff}}$ using the angular diameter $\theta$.

${ }^{d}$ One metal-rich giant star.

$e$ Did not find any metallicity determination so decided to adopt $[\mathrm{Fe} / \mathrm{H}]=0$.

\section{Comparison with other temperature scales}

In this section, we compare our temperature scale with other temperature determinations based on different implementations of the IRFM (Alonso et al. 1996b, 1999a; Ramírez \& Meléndez 2005a; Casagrande et al. 2006), on the excitation equilibrium of Fe I lines (Santos et al. 2004), and on the fitting of Balmer line profiles (Barklem et al. 2002; Bonifacio et al. 2007). In Table 4 we gather the mean differences between our temperatures and those of different samples, $\Delta T_{\text {eff }}$, together with the standard deviation (scatter) around the mean, $\sigma_{T_{\text {eff }}}$. 


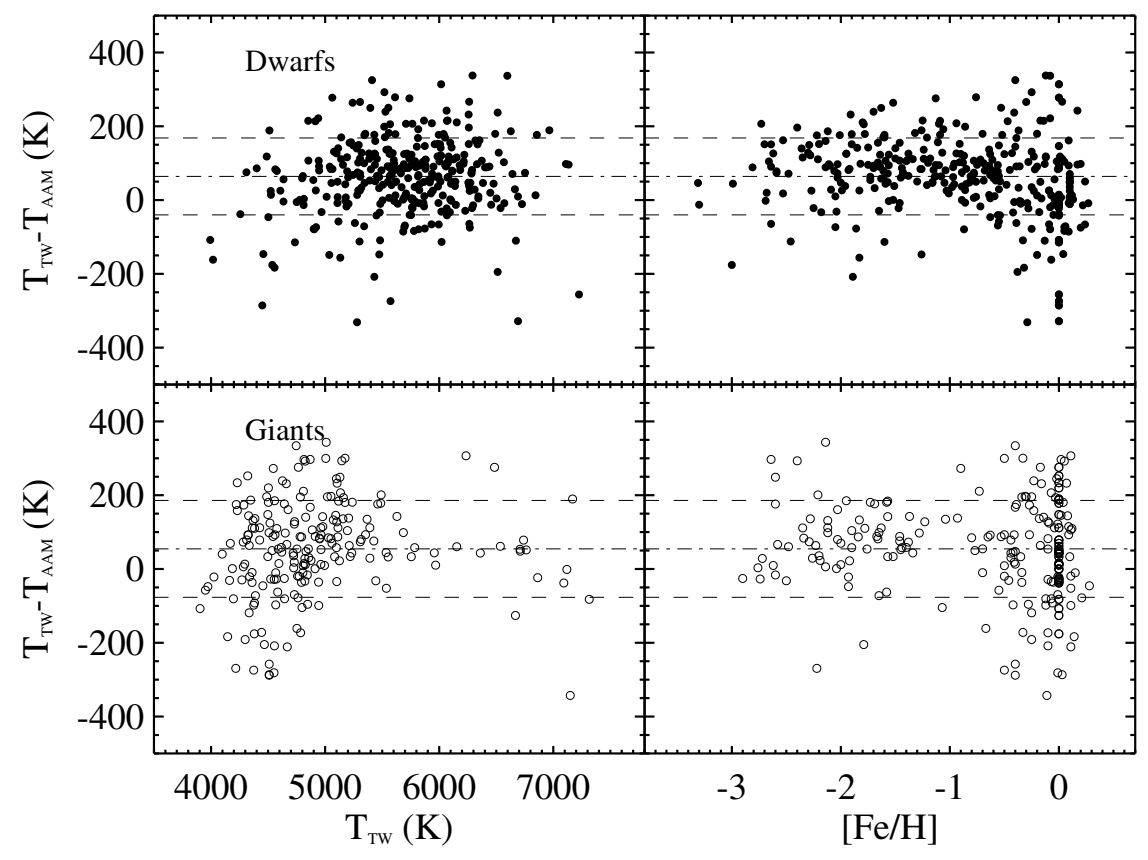

Fig. 4. Comparison of our temperature scale with that of Alonso et al. (1996a, 1999a). The dashed-dotted line indicates the average temperature difference and dashed lines the standard deviation, $1 \sigma$, from the average (see text).

\subsection{Alonso et al. sample}

The updated temperatures do not differ significantly from those of Alonso et al. (1996a, 1999a, see Fig. 4). Our temperature scale is hotter than that of Alonso et al. (1996a, 1999a) for both dwarfs and giants. We find an average difference $\Delta T_{\text {eff }}=+64 \mathrm{~K}$ with a $\sigma_{T_{\text {eff }}}=104 \mathrm{~K}(N=332 \mathrm{dwarfs})$ and $\Delta T_{\text {eff }}=+54 \mathrm{~K}$ with a $\sigma_{T_{\text {eff }}}=131 \mathrm{~K}(N=202$ giants $)$. This translates into a mean $T_{\text {eff }}$ difference of $\lesssim 1 \%$. Although not negligible, such differences are within the error bars of the current temperature determinations, although the scatter, $\sigma_{T_{\text {eff }}}$, seems to be quite large. The different bolometric flux calibration, photometric data and absolute flux calibration might be responsible for this small difference between the two temperature scales. Casagrande et al. (2006) checked that using the absolute calibration of Alonso et al. (1995) and if using the TCS filters, their calibration and that of Ramírez \& Meléndez (2005a) agree within $20 \mathrm{~K}$. However, to do this exercise they had to transform the 2MASS magnitudes into the TCS system, so their conclusions may be affected by these transformations.

Even if we select subsamples of different mean metallicity, the differences remain very small (see Table 4 ). In conclusion, in the whole metallicity range, the systematic difference between our temperature scale and that of Alonso et al. (1996a, 1999a) in dwarfs and giants is positive but smaller than $+65 \mathrm{~K}$, which is in fact less that the average of the individual uncertainties in our calibration ( $\$ 82 \mathrm{~K}$ for dwarfs and $\lesssim 76 \mathrm{~K}$ for giants).

\subsection{Ramírez \& Meléndez sample}

Ramírez \& Meléndez (2005a) extend the sample of stars of Alonso et al. (1996a, 1999a) with metal-rich stars from Santos et al. (2004) and very metal-poor stars from Christlieb et al. (2004) and Cayrel et al. (2004). We determined effective temperatures for the calibrators of Ramírez \& Meléndez (2005a) using our implementation of the IRFM. Our temperature scale is on average hotter than that of Ramírez \& Meléndez (2005a) by about
$\Delta T_{\text {eff }}=+33 \mathrm{~K}\left(\sigma_{T_{\text {eff }}}=98 \mathrm{~K}, N=84 \mathrm{dwarfs}\right)$ and $\Delta T_{\text {eff }}=+63 \mathrm{~K}$ $\left(\sigma_{T_{\text {eff }}}=57 \mathrm{~K}, N=25\right.$ giants). This difference might be partially related to the use of different absolute calibration as we stated in Sect. 8.1.

Among giants, we find minor differences when we look at the most metal-poor and metal-rich stars in the sample (see Table 4). However, this behaviour changes when we inspect the dwarf stars. While for metal-rich dwarfs we find $\Delta T_{\text {eff }}=+45 \mathrm{~K}$ $\left(\sigma_{T_{\text {eff }}}=76 \mathrm{~K}, N=69\right.$ dwarfs with $\left.[\mathrm{Fe} / \mathrm{H}]>-0.5\right)$, for metalpoor dwarfs we find our temperature scale to be cooler: $\Delta T_{\text {eff }}=$ $-87 \mathrm{~K}\left(\sigma_{T_{\text {eff }}}=194 \mathrm{~K}, N=12\right.$ dwarfs with $\left.[\mathrm{Fe} / \mathrm{H}]<-2.5\right)$. We believe that this difference is mainly due to the photometric transformations between the 2MASS and the TCS systems that Ramírez \& Meléndez (2005a) need to perform in order to derive the IRFM temperatures.

\subsection{Casagrande et al. sample}

Casagrande et al. (2006) propose a new IRFM using multiband photometry. They derive empirical effective temperature and bolometric flux calibration for $\mathrm{G}$ and $\mathrm{K}$ dwarfs stars in the range $-1.87<[\mathrm{Fe} / \mathrm{H}]<0.34$. They use $B V(R I)_{\mathrm{C}}$ Johnson-Cousins photometry and $J H K_{\mathrm{S}} 2 \mathrm{MASS}$ photometry. We applied our implementation to estimating the $T_{\text {eff }}$ of stars in their sample and find our temperature scale only slightly cooler by $\Delta T_{\text {eff }}=-12 \mathrm{~K}$ $\left(\sigma_{T_{\mathrm{eff}}}=56 \mathrm{~K}, N=101 \mathrm{dwarfs}\right)$. For these stars, we estimated the reddening corrections from the maps of dust of Schlegel et al. (1998), corrected as described in Sect. 4.3. In Table 4, we also compare our temperature scale with that of Casagrande et al. (2006) by arbitrarily adopting the reddening corrections $E(B-V)=0$ for all the stars, under the assumption that all these stars are nearby and should not show any reddening at all. We find $\Delta T_{\mathrm{eff}}=-41 \mathrm{~K}\left(\sigma_{T_{\mathrm{eff}}}=50 \mathrm{~K}, N=101 \mathrm{dwarfs}\right)$, i.e. temperatures $29 \mathrm{~K}$ cooler on average. This systematic difference is well within our error budget, so we decided to use these reddening corrections to be consistent with other new stars included in the 
sample for which we need to estimate the reddening corrections as the dwarf stars from Bonifacio et al. (2007, see Sect. 8.6).

\subsection{Santos et al. sample}

Santos et al. (2004) have carried out a detailed spectroscopic analysis of planet-host stars and a comparison sample of stars without known planets. Their effective temperatures are based on the excitation equilibrium of the Fe I lines. Our temperature scale is only slightly hotter than that of Santos et al. (2004) with $\Delta T_{\text {eff }}=+11 \mathrm{~K}\left(\sigma_{T_{\text {eff }}}=120 \mathrm{~K}, N=133 \mathrm{dwarfs}\right)$, although with a large scatter. As in the previous section, we also derived the reddening corrections from the Schlegel et al. (1998) dust maps, corrected as described in Sect. 4.3. In Table 4, we also show the comparison with $E(B-V)=0$.

\subsection{Cayrel et al. sample}

Cayrel et al. (2004) present UVES spectroscopic observations of very metal-poor giant stars. They derived $T_{\text {eff }}$ using the $T_{\text {eff }}$ :colour- $[\mathrm{Fe} / \mathrm{H}]$ calibrations of Alonso et al. (1999b). Our $T_{\text {eff }}$ scale is hotter by $\Delta T_{\text {eff }}=+115 \mathrm{~K}\left(\sigma_{T_{\text {eff }}}=76 \mathrm{~K}, N=34\right.$ giants with $[\mathrm{Fe} / \mathrm{H}]<-2.5)$; however, that part of this difference stems from the different choice made for the reddening. Here we adopted the reddening from the dust maps of Schlegel et al. (1998), corrected as described in Sect. 4.3, whereas Cayrel et al. (2004) instead used the Burstein \& Heiles (1982) maps. The different choice in reddening accounts for a difference of $\sim 40-50 \mathrm{~K}$, on average (Cayrel et al. 2004). The remaining $75 \mathrm{~K}$ reflect the difference between our calibration and that of Alonso et al. (1999b). Unsurprisingly, this is, essentially, the same as what was found for giant stars with $[\mathrm{Fe} / \mathrm{H}]<-2.5$ in Sect. 8.1 (see Table 4).

\subsection{Bonifacio et al. sample}

Bonifacio et al. (2007) present high quality spectroscopic data of a sample of extremely metal-poor dwarf stars. They derived the effective temperatures by fitting the wings of the $\mathrm{H} \alpha$ line. We derived the effective temperatures of these stars using the 2MASS $J H K_{\mathrm{s}}$ magnitudes and reddenings from Schlegel et al. (1998), corrected as described in Sect. 4.3. Our effective temperatures are significantly hotter than those derived from the Balmer lines, $\Delta T_{\text {eff }}=+165 \mathrm{~K}\left(\sigma_{T_{\text {eff }}}=79 \mathrm{~K}, N=16\right.$ dwarfs with $[\mathrm{Fe} / \mathrm{H}]<-2.5)$. The difference between the temperatures derived from $\mathrm{H} \alpha$, and those derived by using the colour $V-K$ in the calibrations of Ramírez \& Meléndez (2005b) is roughly $265 \pm 122 \mathrm{~K}$. This $\sim 100 \mathrm{~K}$ difference may be partially explained by our comparison with the temperature scale of Ramírez \& Meléndez (2005a) with $\Delta T_{\text {eff }}=-87 \mathrm{~K}$ (see Sect. 8.2). This has an impact on the Li abundances in extremely metal-poor stars down to $[\mathrm{Fe} / \mathrm{H}]=-4$ (see Sbordone et al. 2008, in prep.), because the IRFM temperatures would provide higher $\mathrm{Li}$ abundances at the lowest metallicities, whereas $\mathrm{H} \alpha$ temperatures seem to show a slowly decreasing trend in Li towards lower metallicities.

Bonifacio et al. (2007) use the theory of Barklem et al. (2000) to describe the self-broadening of Balmer lines. For the same sample of stars, Bonifacio et al. (2003) instead use the Ali $\&$ Griem $(1965,1966)$ theory and derived effective temperatures which were on average $150 \mathrm{~K}$ hotter, thus in substantial agreement with our IRFM temperatures.

\subsection{Christlieb et al. sample}

Christlieb et al. (2004) present the Hamburg/ESO R-process Enhanced Star survey (HERES) with the aim of searching for very metal-poor stars $([\mathrm{Fe} / \mathrm{H}]<-2.5)$ with $r$-process elements enhanced. We selected those stars with available $B-V$ and $V$ photometry in Christlieb et al. (2004) and took the stellar parameters from Barklem et al. (2005). The effective temperatures were estimated by averaging the resulting $T_{\text {eff }}$ from the different $T_{\text {eff }}$ :colour-[Fe/H] calibrations of Alonso et al. (1996b) and Alonso et al. (1999b). They followed the prescription described by Sivarani et al. (2004). Our $T_{\text {eff }}$ scale is significantly hotter by $\Delta T_{\text {eff }}=+177 \mathrm{~K}\left(\sigma_{T_{\text {eff }}}=80 \mathrm{~K}, N=8\right.$ dwarfs with $-3.1[\mathrm{Fe} / \mathrm{H}]<-1.6)$ and $\Delta T_{\text {eff }}=+128 \mathrm{~K}\left(\sigma_{T_{\text {eff }}}=71 \mathrm{~K}, N=22\right.$ giants with $-3.4[\mathrm{Fe} / \mathrm{H}]<-2.6)$. This difference probably comes from the different adopted reddenings and the difference between our temperature scale and that of Alonso et al. (1996a) and Alonso et al. (1999a).

\subsection{Barklem et al. sample}

Balmer-line profile fitting in principle allows a very precise determination of stellar effective temperature for cool stars. Barklem et al. (2002) claim an accuracy of the temperature determinations of $\sim 65 \mathrm{~K}$ for solar metallicity stars but for $[\mathrm{Fe} / \mathrm{H}] \sim-1$ of $\sim 80$ and $[\mathrm{Fe} / \mathrm{H}] \sim-2$ of $\sim 100 \mathrm{~K}$. Uncertainties in the theory of self-broadening, deviations from LTE and granulation effects add to the systematic error budget of Balmer-line based effective temperatures. We compared our temperature scale with that of Barklem et al. (2002). Our temperatures are hotter by $\Delta T_{\text {eff }}=$ $+77 \mathrm{~K}\left(\sigma_{T_{\text {eff }}}=133 \mathrm{~K}, N=23\right.$ dwarfs with $\left.[\mathrm{Fe} / \mathrm{H}]>-2.5\right)$. However, for metal-rich dwarfs with $[\mathrm{Fe} / \mathrm{H}]>-0.5$, this difference drops to $\Delta T_{\text {eff }}=+51 \mathrm{~K}\left(\sigma_{T_{\text {eff }}}=129 \mathrm{~K}, N=16\right.$ dwarfs with $[\mathrm{Fe} / \mathrm{H}]>-0.5)$. The average difference between the two temperature scales remains within the uncertainties on the temperature determinations, although the standard deviation is large.

\section{9. $T_{\text {eff }}:$ colour $-[\mathrm{Fe} / \mathrm{H}]$ calibrations}

We derived relations between $T_{\text {eff }}$ and colours, also taking the effects of metallicity into account. We adopted the same fitting formula that was adopted by Alonso et al. (1996b, 1999b), Ramírez \& Meléndez (2005b), and Casagrande et al. (2006)

$\theta_{\mathrm{eff}}=b_{0}+b_{1} X+b_{2} X^{2}+b_{3} X[\mathrm{Fe} / \mathrm{H}]+b_{4}[\mathrm{Fe} / \mathrm{H}]+b_{5}[\mathrm{Fe} / \mathrm{H}]^{2}(10)$

where $\theta_{\mathrm{eff}}=5040 / T_{\mathrm{eff}}, X$ represents the colour, and $b_{i}(i=$ $0, \ldots, 5)$ are the coefficients of the fit. We iterate the fitting procedure by discarding the points more than $2.5 \sigma$ from the mean fit. All our calibrations were adequately tested by removing some terms and/or adding higher order terms in either $X$ and $[\mathrm{Fe} / \mathrm{H}]$. We verified that neither removing terms nor introducing higher order terms improves the accuracy of the fit significantly. Therefore we adopted Eq. (10).

In Figs. 5-8, we display the polynomial fits which represent the empirical calibrations $T_{\text {eff }}$ versus colours and metallicity. We discarded all the stars with uncertainties in the $J H K_{\mathrm{s}}$ magnitudes greater than 0.1 . The coefficient of these calibrations are given in Table 5, along with the remaining number of stars after the $2.5 \sigma$ clipping and the rms of the fit, $\sigma\left(T_{\text {eff }}\right)$. Normally, the number of iterations were fewer than 20. Our polynomial fits of the colour $B-V$ usually have similar rms. than those provided by Ramírez $\&$ Meléndez (2005b). We should point out the small number of giant stars with metallicities $[\mathrm{Fe} / \mathrm{H}]>-1.5$, because most of the 

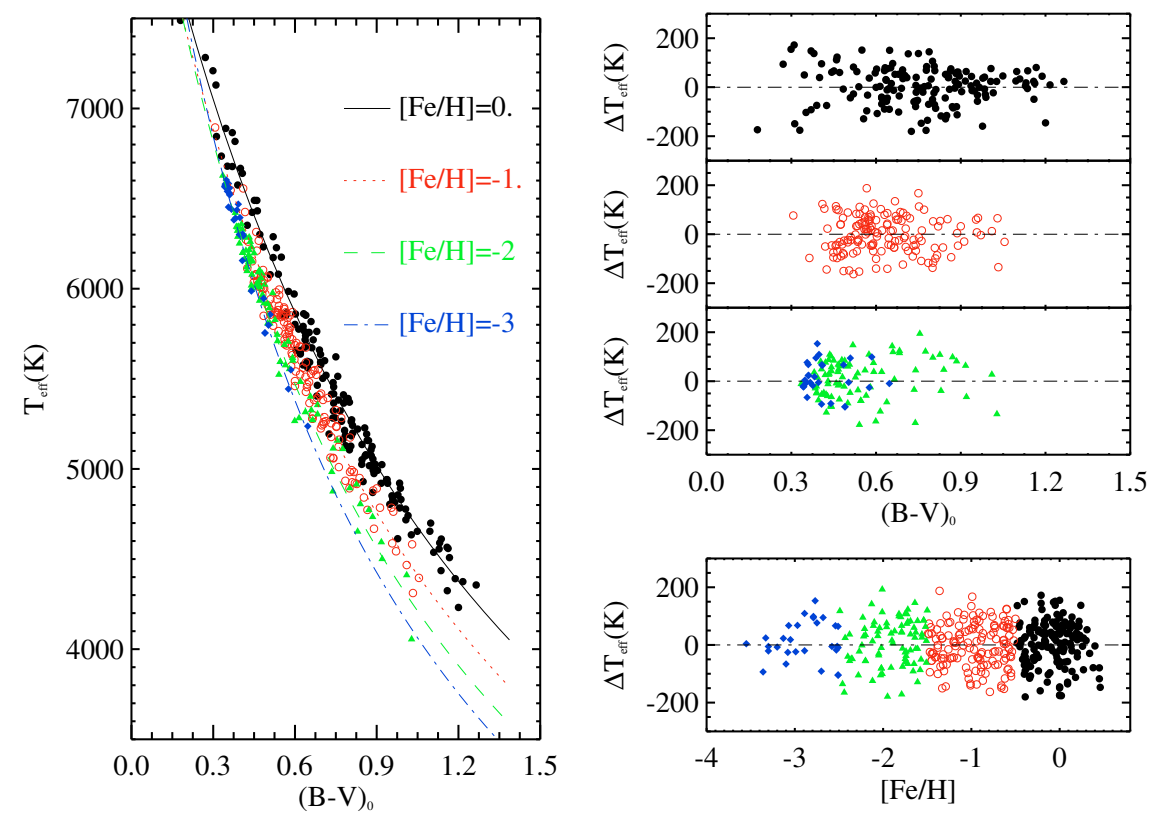

Fig. 5. Left: empirical calibration $T_{\text {eff }}:(B-V)-[\mathrm{Fe} / \mathrm{H}]$ for dwarfs in the metallicity bins $-0.5<[\mathrm{Fe} / \mathrm{H}] \leq 0.5$ (filled circles), $-1.5<[\mathrm{Fe} / \mathrm{H}] \leq-0.5$ (open circles), $-2.5<[\mathrm{Fe} / \mathrm{H}] \leq-1.5$ (triangles), and $[\mathrm{Fe} / \mathrm{H}] \leq-2.5$ (diamonds). The lines correspond to our calibration for $[\mathrm{Fe} / \mathrm{H}]=0$ (solid line), -1.0 (dotted line), -2.0 (dashed line), -3.0 (dotted-dashed line). Right: residuals of the fit $\left(\Delta T_{\mathrm{eff}}=T_{\mathrm{eff}}^{\mathrm{IRFM}}-T_{\mathrm{eff}}^{\mathrm{cal}}\right)$ as a function of $(B-V)$.
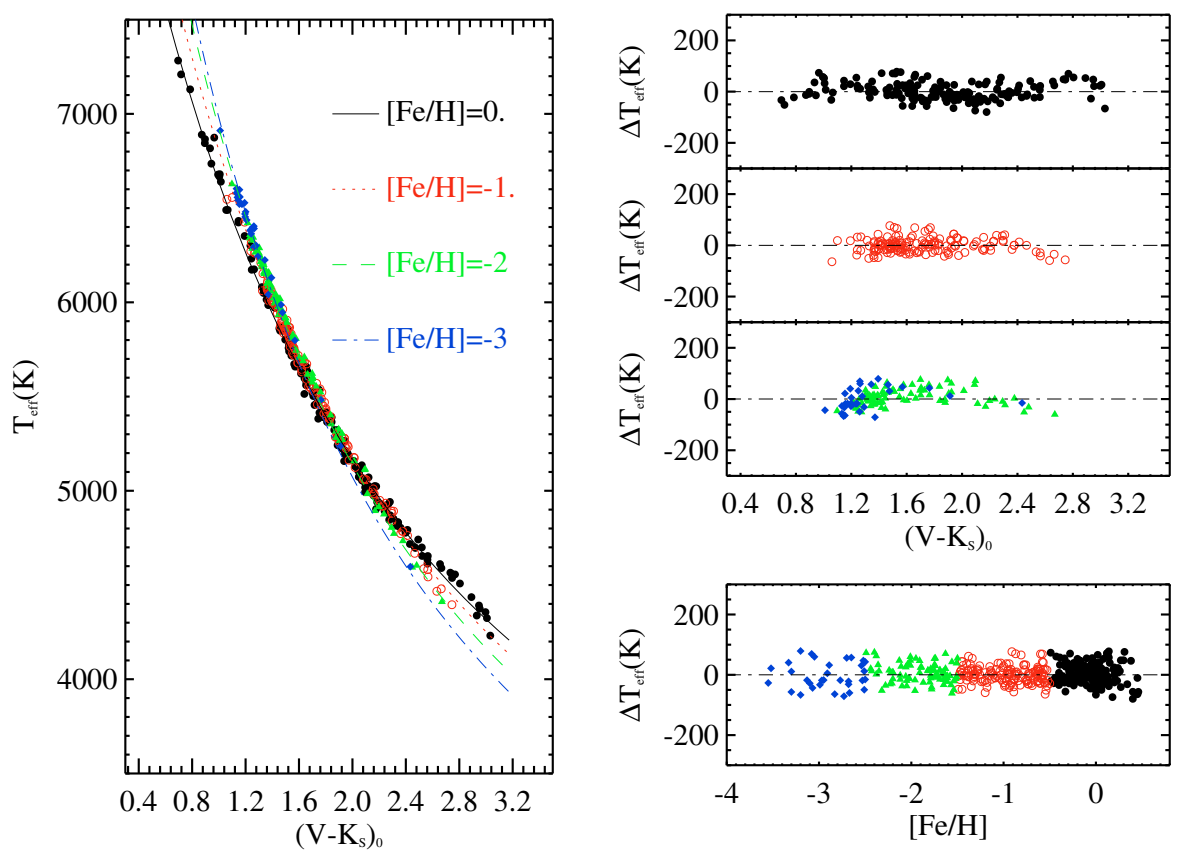

Fig. 6. The same as Fig. 5, but for $V-K_{\mathrm{s}}$ in dwarfs.

giant stars in the sample of Alonso et al. (1999a) are very bright objects, hence with poor-quality $2 \mathrm{MASS} J H K_{\mathrm{S}}$ magnitudes. For dwarf stars, our empirical calibrations of $V-R$ and $V-I$ have smaller rms than those of Ramírez \& Meléndez (2005b). For giant stars, the calibration of $V-R$ shows a greater rms than in Ramírez \& Meléndez (2005b), probably due to the small number of stars in our sample.

On the other hand, our empirical calibrations of the colours $V-J, V-H$, and $V-K_{\mathrm{s}}$ have a smaller rms than those presented by Ramírez \& Meléndez (2005b). For giants, our fits are more accurate although our sample contains slightly fewer giant stars than the sample of Ramírez \& Meléndez (2005b).

\section{Angular diameters}

The IRFM was developed to provide the $T_{\mathrm{eff}}$ and $\theta$ simultaneously from observed and theoretical data. One fundamental test to the IRFM is thus the comparison with measured angular diameters. Ramírez \& Meléndez (2005a) compare their results with measured angular diameters from Richichi \& Percheron (2002) and Kervella et al. (2004) for giant and dwarf stars, respectively. These stars are too bright for the 2MASS catalogue, making the 2MASS $J H K_{\mathrm{s}}$ magnitudes very uncertain. However, Baines et al. (2008) have recently presented new measurements of angular diameters using the Center for High Angular Resolution 

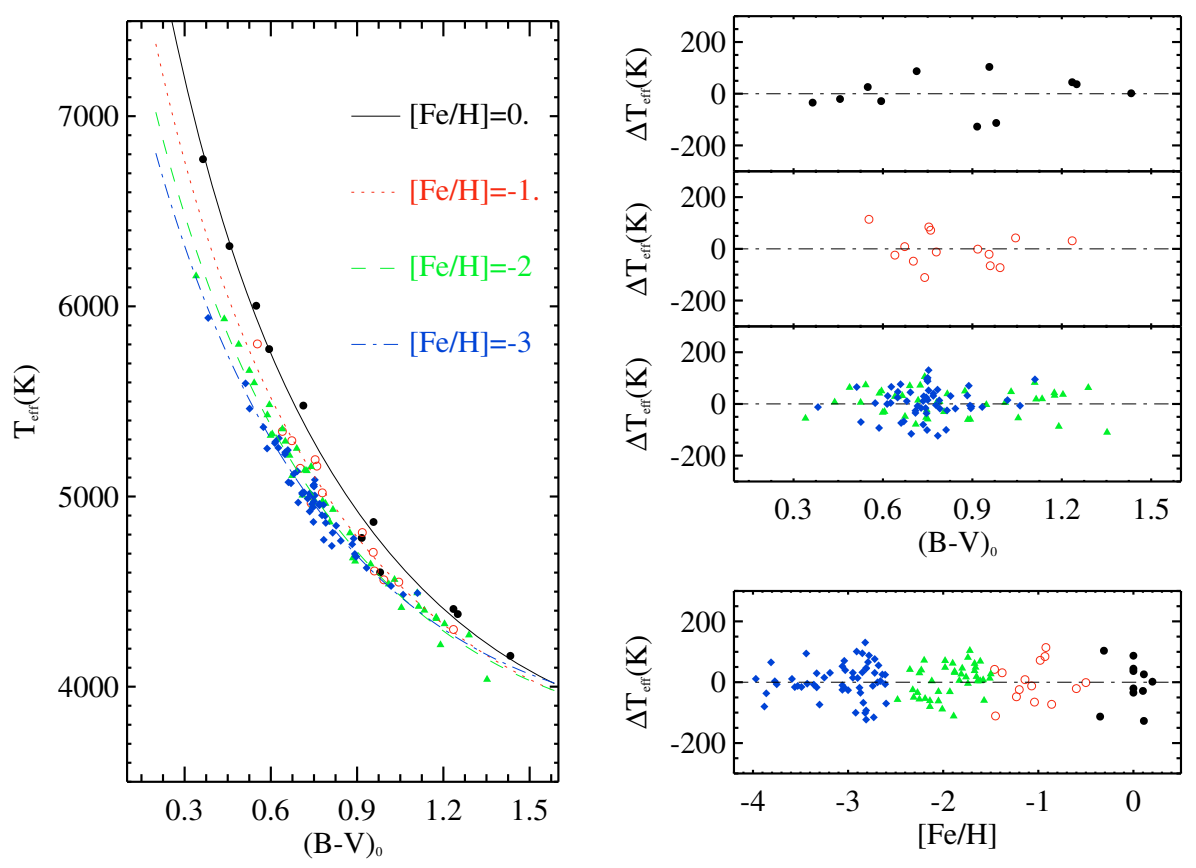

Fig. 7. The same as Fig. 5, but for giants.
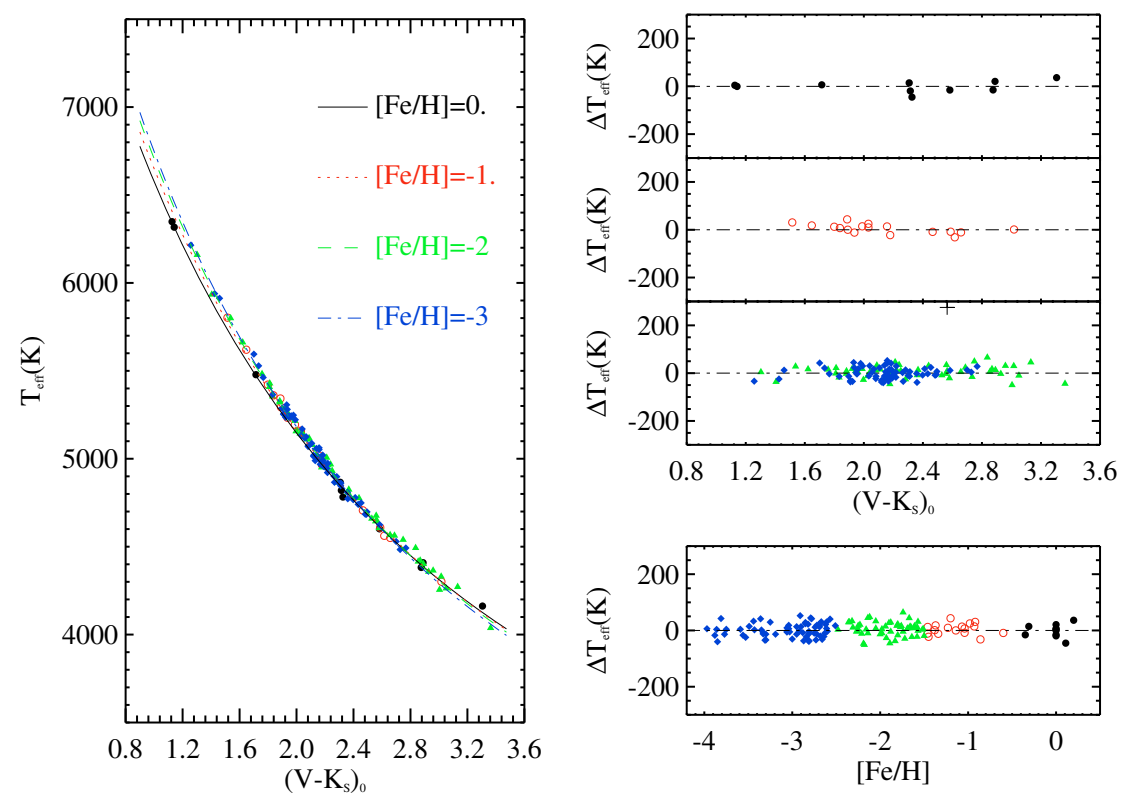

Fig. 8. The same as Fig. 5, but for $V-K_{\mathrm{s}}$ in giants.

Astronomy (CHARA) Array, a six-element Y-shaped interferometric array. We searched for the $J H K_{\mathrm{S}}$ magnitudes of the stars reported in Baines et al. (2008) and the results are presented in Figs. 9 and 10. The stellar parameters were adopted from Baines et al. (2008), and the metallicities were extracted from Santos et al. (2004) and from Cayrel de Strobel et al. (2001). However, for same cases, especially for giant stars, we did not find any available metallicity determination, so we decided to adopt $[\mathrm{Fe} / \mathrm{H}]=0$. The $V$ magnitudes were extracted from the GCPD (Mermilliod et al. 1997), and in those cases where no value was found, we took the $V$ magnitude as given in the SIMBAD catalogue. One can compare our IRFM angular diameters, $\theta_{\mathrm{IRFM}}$, with the direct measurements, $\theta_{\mathrm{LD}}$. For dwarf stars, the average difference, $\Delta \theta=\theta_{\mathrm{IRFM}}-\theta_{\mathrm{LD}}$ is 0.002 with a standard deviation $\sigma_{\theta}=0.033(N=22$ stars $)$. We can also derive a direct temperature, $T_{\mathrm{eff}}^{\mathrm{dir}}$, from $\theta_{\mathrm{LD}}$ and the bolometric flux, determined from our bolometric flux calibration, by using Eq. (1). The previous comparison between angular diameters thus translates into a temperature difference, $\Delta T_{\mathrm{eff}}=T_{\mathrm{eff}}^{\mathrm{IRF}}-T_{\mathrm{eff}}^{\mathrm{dir}}$, of -32 with $\sigma_{T_{\text {eff }}}=163$ ( $N=22$ stars). For giants, the number of stars with relatively accurate $J H K_{\mathrm{s}}$ data is low. The sample of Baines et al. (2008) contains only six giant stars. For these stars, we find $\Delta \theta=0.012$ with a $\sigma_{\theta}=0.029$ which translates into $\Delta T_{\text {eff }}=-67$ with a $\sigma_{T_{\text {eff }}}=139$. These results are also given in Table 4 in comparison with other temperature determinations. Our new implementation of the IRFM provides good results when comparing 
Table 5. Coefficients and range of applicability of the $T_{\text {eff }}:$ colour $-[\mathrm{Fe} / \mathrm{H}]$ calibrations.

\begin{tabular}{lccrrrrrrrr}
\hline \hline Colour & Colour range & {$[\mathrm{Fe} / \mathrm{H}]$ range } & $b_{0}$ & $b_{1}$ & $b_{2}$ & $b_{3}$ & $b_{4}$ & $b_{5}$ & $\mathrm{~N}^{a}$ & $\sigma_{T_{\mathrm{eff}}}(\mathrm{K})^{b}$ \\
\hline & & & \multicolumn{7}{c}{ Dwarf stars } \\
$B-V$ & {$[0.2,1.3]$} & {$[-3.5,0.5]$} & 0.5725 & 0.4722 & 0.0086 & -0.0628 & -0.0038 & -0.0051 & 418 & 76 \\
$V-R$ & {$[0.2,0.8]$} & {$[-3.1,0.3]$} & 0.4451 & 1.4561 & -0.6893 & -0.0944 & 0.0161 & -0.0038 & 164 & 45 \\
$V-I$ & {$[0.5,1.4]$} & {$[-3.1,0.3]$} & 0.4025 & 0.8324 & -0.2041 & -0.0555 & 0.0410 & -0.0003 & 164 & 52 \\
$V-J$ & {$[0.5,2.3]$} & {$[-3.5,0.5]$} & 0.4997 & 0.3504 & -0.0230 & -0.0295 & 0.0468 & 0.0037 & 430 & 36 \\
$V-H$ & {$[0.6,2.8]$} & {$[-3.5,0.5]$} & 0.5341 & 0.2517 & -0.0100 & -0.0236 & 0.0523 & 0.0044 & 426 & 30 \\
$V-K_{\mathrm{s}}$ & {$[0.7,3.0]$} & {$[-3.5,0.5]$} & 0.5201 & 0.2511 & -0.0118 & -0.0186 & 0.0408 & 0.0033 & 431 & 32 \\
$J-K_{\mathrm{s}}$ & {$[0.1,0.8]$} & {$[-3.5,0.5]$} & 0.6524 & 0.5813 & 0.1225 & -0.0646 & 0.0370 & 0.0016 & 436 & 139 \\
\hline & & & \multicolumn{7}{c}{ Giant stars } \\
$B-V$ & {$[0.3,1.4]$} & {$[-4.0,0.2]$} & 0.4967 & 0.7260 & -0.1563 & 0.0255 & -0.0585 & -0.0061 & 120 & 57 \\
$V-R$ & {$[0.3,0.7]$} & {$[-4.0,0.1]$} & 0.4530 & 1.4347 & -0.5883 & -0.0156 & -0.0096 & -0.0039 & 55 & 85 \\
$V-J$ & {$[1.0,2.4]$} & {$[-4.0,0.2]$} & 0.4629 & 0.4124 & -0.0417 & -0.0012 & 0.0094 & 0.0013 & 138 & 18 \\
$V-H$ & {$[0.8,3.1]$} & {$[-4.0,0.2]$} & 0.5321 & 0.2649 & -0.0146 & -0.0069 & 0.0211 & 0.0009 & 144 & 23 \\
$V-K_{\mathrm{s}}$ & {$[1.1,3.4]$} & {$[-4.0,0.2]$} & 0.5293 & 0.2489 & -0.0119 & -0.0042 & 0.0135 & 0.0010 & 145 & 23 \\
$J-K_{\mathrm{s}}$ & {$[0.1,0.9]$} & {$[-4.0,0.2]$} & 0.6517 & 0.6312 & 0.0168 & -0.0381 & 0.0256 & 0.0013 & 145 & 94 \\
\hline
\end{tabular}

${ }^{a}$ The remaining number of stars after several iterations (usually less than 20 ) of the $2.5 \sigma$ clipping. ${ }^{b} \sigma_{T_{\text {eff }}}$, given in $\mathrm{K}$, is the standard deviation of the final calibrations.
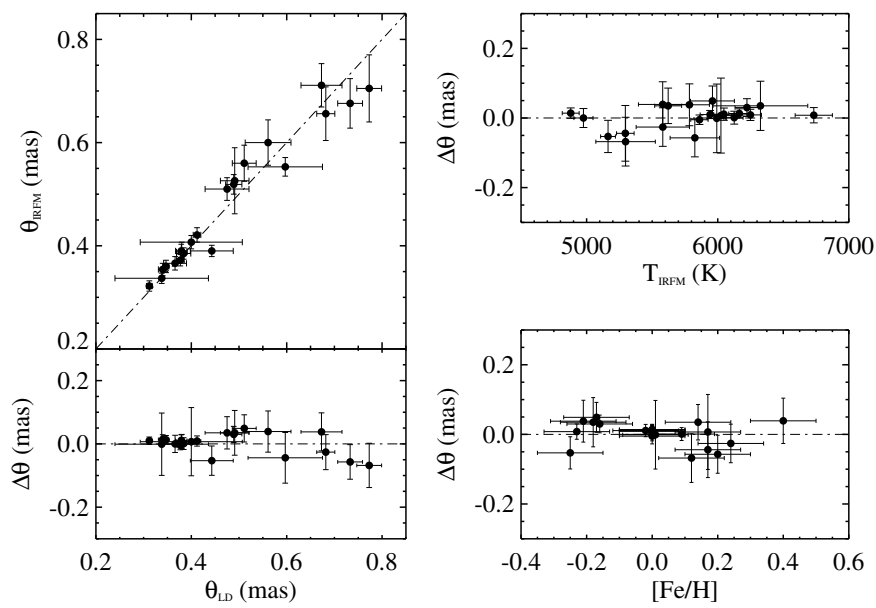

Fig. 9. Comparison between the angular diameters from Baines et al. (2008), $\theta_{\mathrm{LD}}$, and diameters from the IRFM, $\theta_{\mathrm{IRFM}}$ for dwarf stars. Error bars are individual uncertaintes. The differences between $\theta_{\mathrm{IRFM}}$ and $\theta_{\mathrm{LD}}$, $\Delta \theta$, are also shown containing the uncertainties of both $\theta_{\mathrm{IRFM}}$ and $\theta_{\mathrm{LD}}$.

with direct measurements of angular diameters. The absence of any trend with metallicity in the residuals shown in Fig. 9 over almost 1 dex in metallicity is very encouraging. This suggests that the model atmospheres correctly model the variation of fluxes with metallicity. Since the metal-rich range is the most difficult for modelling the opacity, it is reasonable to expect that the models are also reliable at low metallicity. In other words, we do not expect that our temperature scale has spurious trends with metallicity due to inadequate modelling of the stellar atmospheres.

\section{Summary}

We have made use of the IRFM to determine effective temperatures of 555 dwarf and subgiant field stars and of 264 giant field stars. Our implementation of the IRFM uses the 2MASS photometric system as a reference system to perform all the calculations. We derived a bolometric flux calibration down to metallicities of $[\mathrm{Fe} / \mathrm{H}]=-3.5$ for dwarfs and -4.0 for giants, as a function of the 2MASS magnitude, $m$, and the colour, $V-m$.
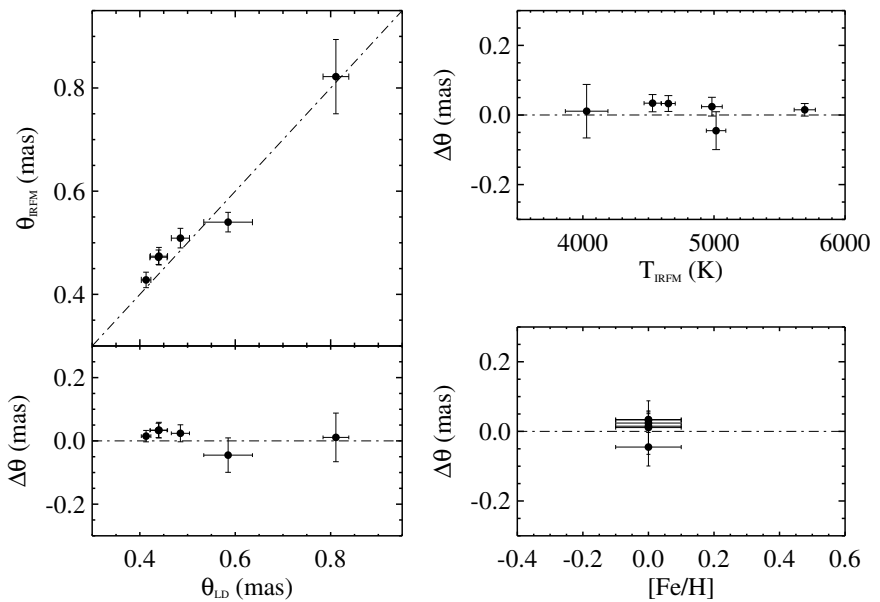

Fig. 10. Same as Fig. 9, but for giants.

We computed theoretical magnitudes by integrating the ATLAS models in the 2MASS $J H K_{\mathrm{s}}$ filters.

Our temperature scale is hotter than that of Alonso et al. (1996a, 1999a) by $\sim 64 \mathrm{~K}\left(\sigma_{\mathrm{T}}=104 \mathrm{~K}, N=332\right.$ dwarfs) and $\sim 54 \mathrm{~K}\left(\sigma_{\mathrm{T}}=131 \mathrm{~K}, N=202\right.$ giants $)$. Similar results are found when comparing with Ramírez \& Meléndez (2005a). However, interestingly, for dwarfs stars with $[\mathrm{Fe} / \mathrm{H}]<-2.5$, the comparison with the sample of Ramírez \& Meléndez (2005a) provides a difference of $\sim-87 \mathrm{~K}\left(\sigma_{\mathrm{T}}=194 \mathrm{~K}, N=12\right.$ dwarfs $)$. We believe this difference is related to Ramírez \& Meléndez (2005a) transforming the 2MASS $J H K_{\mathrm{S}}$ magnitudes to the TCS photometric system to derive bolometric fluxes and effective temperatures for their calibrators at the lowest metallicities, whereas we determine the bolometric fluxes and effective temperatures in the 2MASS photometric system.

Our $T_{\text {eff }}$ are hotter than those estimated using $H \alpha$ line profiles by $\Delta T_{\text {eff }}=+77 \mathrm{~K}$ (Barklem et al. $2002, \sigma_{T_{\text {eff }}}=133 \mathrm{~K}$, $N=23$ dwarfs with $[\mathrm{Fe} / \mathrm{H}]>-2.5)$ and $\Delta T_{\text {eff }}=+165 \mathrm{~K}$ (Bonifacio et al. 2007, $\sigma_{T_{\text {eff }}}=79 \mathrm{~K}, N=16$ dwarfs with $[\mathrm{Fe} / \mathrm{H}]<-2.5)$. This result has implications for the Li abundances for very metal-poor stars down to $[\mathrm{Fe} / \mathrm{H}]=-4$. Higher temperatures provide higher Li abundances; therefore, the drop 
of the $\mathrm{Li}$ abundances towards lower metallicities will cancel out, and the Li abundances would remain in a plateau.

We derived $T_{\text {eff }}$ versus colour empirical calibrations, which are compatible with those presented by Alonso et al. (1996b, 1999b), Ramírez \& Meléndez (2005b), Casagrande et al. (2006), within the quoted errors. For those who wish to use 2MASS photometry to estimate effective temperatures for a wide range of metallicities, we recommend our calibration, which were derived within the 2MASS system, rather than the others, which are either based on different systems or on hybrid systems. Our calibrations exploit the excellent internal consistency of the 2MASS photometry and should provide accurate temperatures in a relative sense. In an absolute sense, our calibrations are of the same quality as the other calibrations.

A comparison of IRFM angular diameters with interferometric measurements of angular diameters from Baines et al. (2008) provides good agreement for both dwarf and giant stars. This gives us confidence that our new implementation of the IRFM is reasonable.

Acknowledgements. We are very grateful to Ivan Ramírez \& Jorge Meléndez for kindly providing us with the photometric data that they collected in 2005 for dwarf and giant stars of Alonso et al. We acknowledge support from the EU contract MEXT-CT-2004-014265 (CIFIST). This publication makes use of data products from the Two Micron All Sky Survey, which is a joint project of the University of Massachusetts and the Infrared Processing and Analysis Center/California Institute of Technology, funded by the National Aeronautics and Space Administration and the National Science Foundation. This research has made use of the SIMBAD database, operated at the CDS, Strasbourg, France.

\section{References}

Ali, A. W., \& Griem, H. R. 1965, Phys. Rev., 140, 1044 Ali, A. W., \& Griem, H. R. 1966, Phys. Rev., 144, 366 Alonso, A., Arribas, S., \& Martinez-Roger, C. 1994, A\&A, 282, 684 Alonso, A., Arribas, S., \& Martinez-Roger, C. 1995, A\&A, 297, 197 Alonso, A., Arribas, S., \& Martinez-Roger, C. 1996a, A\&AS, 117, 227 Alonso, A., Arribas, S., \& Martinez-Roger, C. 1996b, A\&A, 313, 873 Alonso, A., Arribas, S., \& Martínez-Roger, C. 1999a, A\&AS, 139, 335 Alonso, A., Arribas, S., \& Martínez-Roger, C. 1999b, A\&AS, 140, 261 Asplund, M. 2005, ARA\&A, 43, 481

Baines, E. K., McAlister, H. A., Brummelaar, T. A. T., et al. 2008, ApJ, 682, 577 Barklem, P. S. 2007, A\&A, 466, 327

Barklem, P. S., Piskunov, N., \& O’Mara, B. J. 2000, A\&A, 363, 1091

Barklem, P. S., Stempels, H. C., Allende Prieto, C., et al. 2002, A\&A, 385, 951

Barklem, P. S., Christlieb, N., Beers, T. C., et al. 2005, A\&A, 439, 129

Bessell, M. S. 1990, PASP, 102, 1181

Bessell, M. S., Castelli, F., \& Plez, B. 1998, A\&A, 333, 231

Blackwell, D. E., \& Petford, A. D. 1991, A\&A, 250, 459

Blackwell, D. E., \& Shallis, M. J. 1977, MNRAS, 180, 177
Blackwell, D. E., Petford, A. D., Arribas, S., Haddock, D. J., \& Selby, M. J. 1990, A\&A, 232, 396

Bonifacio, P., \& Molaro, P. 1997, MNRAS, 285, 847

Bonifacio, P., Monai, S., \& Beers, T. C. 2000a, AJ, 120, 2065

Bonifacio, P., Caffau, E., \& Molaro, P. 2000b, A\&AS, 145, 473

Bonifacio, P., et al. 2003, Elemental Abundances in Old Stars and Damped Lyman- $\alpha$ Systems, 25th meeting of the IAU, Joint Discussion 15, 22 July 2003, Sydney, Australia, ed. P. E. Nissen, M. Pettini, O. Engvold (San Francisco, CA: ASP), Highlights of Astronomy, 13, 581

Bonifacio, P., Molaro, P., \& Sivarani, T. 2007, A\&A, 462, 851

Brown, T. M., Charbonneau, D., Gilliland, R. L., Noyes, R. W., \& Burrows, A. 2001, ApJ, 552, 699

Burstein D., \& Heiles C., 1982, AJ, 87, 1165

Castelli, F., \& Kurucz, R. L. 1994, A\&A, 281, 817

Castelli, F., \& Kurucz, R. L. 2003, in Modelling of Stellar Atmospheres, ed. N. Piskunov, W. W. Weiss, \& D. F. Gray, IAU Symp. 210, A20P [arXiv:astro-ph/0405087v1]

Casagrande, L., Portinari, L., \& Flynn, C. 2006, MNRAS, 373, 13

Cayrel de Strobel, G., Soubiran, C., \& Ralite, N. 2001, A\&A, 373, 159

Cayrel, R., Depagne, E., Spite, M., et al. 2004, A\&A, 416, 1117

Cohen, M., Wheaton, W. A., \& Megeath, S. T. 2003, AJ, 126, 1090

Christlieb, N., Beers, T. C., Barklem, P. S., et al. 2004, A\&A, 428, 1027

Cutri, R. M., et al. 2003, The IRSA 2MASS All-Sky Point Source Catalog, NASA/IPAC Infrared Science Archive, http://www.ipac.caltech.edu/ 2mass/releases/allsky/doc/explsup.html

Fuhrmann, K., Axer, M., \& Gehren, T. 1993, A\&A, 271, 451

Fuhrmann, K., Axer, M., \& Gehren, T. 1994, A\&A, 285, 585

Hayes, D. S. 1985, Calibration of Fundamental Stellar Quantities, 111, 225

Kervella, P., Thévenin, F., Di Folco, E., \& Ségransan, D. 2004, A\&A, 426, 297

Kervella, P., Mérand, A., Pichon, B., et al. 2008, A\&A, 488, 667

Kurucz, R. L. 1993, ATLAS9 Stellar Atmospheres Programs and $2 \mathrm{~km} \mathrm{~s}^{-1}$ Grid, CD-ROM No. 13, Smithsonian Astrophysical Observatory, Cambridge, MA, USA.

Ludwig, H.-G., Behara, N. T., Steffen, M., \& Bonifacio, P. 2009, A\&A, in preparation

Magain, P. 1987, A\&A, 181, 323

McCall, M. L. 2004, AJ, 128, 2144

Meléndez, J., \& Ramírez, I. 2004, ApJ, 615, L33

Mermilliod, J.-C., Mermilliod, M., \& Hauck, B. 1997, A\&AS, 124, 349

Perryman, M. A. C., Lindegren, L., Kovalevsky, J., et al. 1997, A\&A, 323, L49

Ramírez, I., \& Meléndez, J. 2005a, ApJ, 626, 446

Ramírez, I., \& Meléndez, J. 2005b, ApJ, 626, 465

Richichi, A., \& Percheron, I. 2002, A\&A, 386, 492

Ryan, S. G., Beers, T. C., Deliyannis, C. P., \& Thorburn, J. A. 1996, ApJ, 458, 543

Santos, N. C., Israelian, G., \& Mayor, M. 2004, A\&A, 415, 1153

Santos, N. C., Israelian, G., Mayor, M., et al. 2005, A\&A, 437, 1127

Sbordone et al. 2008, A\&A, in preparation

Schlegel, D. J., Finkbeiner, D. P., \& Davis, M. 1998, ApJ, 500, 525

Shchukina, N., \& Trujillo Bueno, J. 2001, ApJ, 550, 970

Shchukina, N. G., Trujillo Bueno, J., \& Asplund, M. 2005, ApJ, 618, 939

Sivarani, T., Bonifacio, P., Molaro, P., et al. 2004, A\&A, 413, 1073

Skrutskie, M. F., Cutri, R. M., Stiening, R., et al. 2006, AJ, 131, 1163

Spergel, D. N., Bean, R., \& Doré, O. A. 2003, ApJS, 148, 175

Spergel, D. N., Verde, L., \& Peiris, H. V. Å. 2007, ApJS, 170, 377

Spite, M., \& Spite, F. 1982, Nature, 297, 483

Spite, F., \& Spite, M. 1982, A\&A, 115, 357

Spite, M., Cayrel, R., Plez, B., et al. 2005, A\&A, 430, 655

Thévenin, F., \& Idiart, T. P. 1999, ApJ, 521, 753 